\title{
STATISTICAL PROPERTIES OF GENERALIZED VIANA MAPS
}

\author{
PAULO VARANDAS
}

\begin{abstract}
We study quadratic skew-products with parameters driven over piecewise expanding and Markov interval maps with countable many inverse branches, a generalization of the class of maps introduced by Viana Vi97. In particular we construct a class of multidimensional non-uniformly expanding attractors that exhibit both critical points and discontinuities and prove existence and uniqueness of an SRB measure with stretched-exponential decay of correlations, stretched-exponential large deviations and satisfying some limit laws. Moreover, generically such maps admit the coexistence of a dense subset of points with negative central Lyapunov exponent together with a full Lebesgue measure subset of points which have positive Lyapunov exponents in all directions. Finally, we discuss the existence of SRB measures for skewproducts associated to hyperbolic parameters by the study of fibered hyperbolic maps.
\end{abstract}

\section{INTRODUCTION}

Since the 1960's, when the concept of uniform hyperbolicity was coined by Smale in [Sm67], a relevant question in dynamical systems is to construct examples that exhibit the hyperbolic features described by the theory. In fact, Hunt and Mackay HM03] proved that uniformly hyperbolic dynamical systems, among which Smale's horseshoe is a paradigmatic example, arise naturally in physical systems. On other direction, simple onedimensional examples arising from populational dynamics led to consider the quadratic family $T_{a}(x)=a x(1-x)$, or equivalently $f_{a}(x)=1-a x^{2}$, that despite the simple formulation presents very rich and complex dynamics. In fact, it follows from pioneering works by Jakobson, Benedicks and Carleson [Jak81, BC85] that there exists a positive Lebesgue measure set $\Delta \subset(0,2]$ such that $f_{a}(x)=1-a x^{2}$ has an absolutely continuous ergodic probability measure with positive Lyapunov exponent for all $a \in \Delta$, ie, is non-uniformly hyperbolic. Later, Graczyk and Świątek [GS97] and Lyubich Lyu97 proved that $f_{a}$ is hyperbolic for an open and dense set of parameters $a \in(0,2]$.

Much more recently, in a major breakthrough, in [KSvS07a] Kozlovski, Shen and van Strien proved that hyperbolicity is open and dense among $C^{k}$ maps of the interval or the circle $(k \geq 1)$. In particular this shows that although persistent, meaning a positive Lebesgue measure phenomenon for

${ }^{0} 2000$ Mathematics Subject classification: 37A30, 37D35, 37H15, 60F10

Date: October 26, 2018.

Key words and phrases. Non-uniform hyperbolicity, Lyapunov exponents, SRB measure. 
parametrized families, one-dimensional examples with robust non-uniform hyperbolicity do not exist.

In a higher dimensional setting the existence of robust non-uniform hyperbolicity was addressed by Viana [Vi97 that introduced a class of $C^{3}$-maps of the cyclinder: they are any small $C^{3}$-perturbations $\varphi$ of the skew-product transformations

$$
\begin{array}{cccc}
\varphi_{\alpha}: & S^{1} \times I & \rightarrow & S^{1} \times I \\
(\theta, x) & \mapsto & \left(g(\theta), f_{\alpha}(\theta, x)\right)
\end{array}
$$

where $g$ is an expanding map of the unit circle $S^{1}$ with $\left|g^{\prime}(\theta)\right| \geq d$ where $d \geq$ 16 and $f_{\alpha}(\theta, x)=a_{0}+\alpha \sin (2 \pi \theta)-x^{2}$ for some small $\alpha>0$ and parameter $a_{0} \in(0,2]$ such that the quadratic map $h(x)=a_{0}-x^{2}$ is of Misiurewicz type. Despite the presence of a critical region, Viana proved that this class of transformations of the cylinder $S^{1} \times \mathbb{R}$ have positive Lyapunov exponents in every direction, that is

$$
\liminf _{n \rightarrow \infty} \frac{1}{n} \log \left\|D \varphi^{n}(\theta, x) v\right\|>0
$$

for Lebesgue almost every $(\theta, x)$ and all $v \in T_{(\theta, x)}\left(S^{1} \times \mathbb{R}\right)$. Building over this, Alves [Al00] proved that there is a unique $\varphi$-invariant probability measure absolutely continuous with respect to Lebesgue. In other words, this class of $C^{3}$-endomorphisms of the cylinder present a robust non-uniform hyperbolicity phenomenon. More recent contributions and extensions include the ones by Gouzel Gou07] on the skew products with curve of neutral fixed points, by Buzzi, Sester and Tsujii BST03 for $C^{\infty}$-perturbations of the skew product $\varphi_{\alpha}$ with a weaker condition $d \geq 2$ and later on by Schnellmann [Sc08] that considered $\beta$-transformations and by Schnellmann, Gao, Shen [Sc09, GS12] considering Misiurewicz-Thurston quadratic maps as the base dynamics. In these results the proof that Lebesgue almost every point has only positive Lyapunov exponents exploits, in the spirit of [Vi97, some weak hyperbolicity condition, namely dominated decomposition.

An important challenge in dynamics is to construct multidimensional attractors with critical behaviour without dominated splittings but persistence of positive Lyapunov exponents in parameter space. It was proposed by Bonatti, Díaz and Viana in BDV05] that such phenomena might occur in a parametrized family $\left.F(x, y)=\left(a(x, y)-x^{2}, b(x, y)-y^{2}\right)\right)$. As mentioned above, important contributions were given in [Sc09, GS12] where the authors proved non-uniform expansion for skew-product of quadratic maps over a Misiurewicz-Thurston quadratic map. Since parameters corresponding to Misiurewicz-Thurston quadratic maps have zero Lebesgue measure in the parameter space then the previous question remains open.

Our purpose in this paper is to study a class of quadratic skew-products over a Markov expanding map of the interval with at most countably many inverse branches: skew-products $\varphi_{\alpha}(\theta, x)=\left(g(\theta), f_{\alpha}(\theta, x)\right)$ with $g$ piecewise expanding Markov map of the unit interval and $f_{\alpha}(\theta, x)=a_{0}+\alpha \sin (2 \pi \theta)-$ $x^{2}$ for some small $\alpha>0$ and parameter $a_{0} \in(0,2]$. One important motivation to consider skew products of quadratic maps over expanding dynamics with infinitely many branches is to understand if the technique of inducing 
can be an useful approach to the previous question since non-uniform expansion is well known to be related with inducing schemes leading to piecewise expanding maps with infinitely many branches. We prove that the behaviour of this class of transformations has different behaviours depending on the parameter $a_{0}$.

In the one hand, if $h(x)=a_{0}-x^{2}$ is a Misiurewicz quadratic map then we overcome the difficulty caused by the presence of critical points and infinitely many invertibility branches to prove the existence of two positive Lyapunov exponents at Lebesgue almost every point, that there exists a unique absolutely continuous invariant measure and that it satisfies good statistical properties. Moreover, we also prove that generically such transformations admit the coexistence of the full Lebesgue measure set of points which have only positive Lyapunov exponents together with a dense set of points with one positive and one negative Lyapunov exponents, a fact that was unkown even in the context of Viana maps in Vi97. On the other hand, if the quadratic map $h(x)=a_{0}-x^{2}$ is hyperbolic then the skew product $\varphi_{\alpha}$ admits an hyperbolic SRB measure, provided that $\alpha$ is small. In fact this will be consequence of a more result for fibered hyperbolic polynomials.

The difficulties in proving our results are substantially different depending if one is considers perturbations of a Misiurewicz or a hyperbolic quadratic map. In the first case, the strategy of [Vi97] for the positivity of Lyapunov exponents Lebesgue almost everywhere is to consider the iteration of admissible curves (a class of curves preserved under iteration) and to prove that Lebesgue almost every point in each admissible curve has a slow recurrence condition to the critical region and thus has exponential growth of the derivative. The key combinatorial argument in [Vi97 does not hold in our setting since any admissible curve is mapped onto countably many of them. In our Proposition 4.1 we overcome this difficulty and obtain a statistical argument showing that, up to consider a finite iteration $\varphi^{N}$ of the skew-product $\varphi$, the proportion of points in any admissible curve whose iterate intersects a small neighborhood of the critical region is bounded by the Lebesgue measure of the corresponding neighborhood. Then, a large deviations argument shows that the measure of points in admissible curves that exhibit fast recurrence to a neighborhood of the critical region decrease subexponentially and, consequently, Lebesgue almost every point has two positive Lyapunov exponents. In the second case, corresponding to perturbation of hyperbolic quadratic maps, the problem can be understood as a random composition of nearby quadratic maps and so we consider a more general setting of fibered nearby hyperbolic interval maps. The hyperbolicity of the Julia set for fibered polynomial maps in the complex variable setting has been studied by Jonsson [Jo97] and Sester [Se99. Here we use a small neighborhood of the periodic atracting orbit to prove that there exists a trapping region and an attractor. The transversality of admissible curves implies that the attractor does not coincide with the continuation of the periodic attracting orbits, but using a uniforme contraction property one proves that it still supports a unique hyperbolic SRB measure. Roughly, the complement of the basin of attraction is formed by points whose random iteration of quadratic maps that does not intersect the trapping region. 
This paper is organized as follows. In Section 2 we recall some definitions and state our main results. Some preliminary results on one-dimensional dynamics and admissible curves are given along Section 3. Finally, the proofs of the main results are given on Sections 4 , 5 and 6 .

\section{Statement of Main Results}

In this section we present the necessary definitions to state our main results.

2.1. Setting. Let $\mathcal{P}=\left\{\omega_{i}\right\}_{i \in S, S \subset \mathbb{N}_{0}}$ be an at most countable partition of the unit interval $(0,1]$ by subintervals and $g:(0,1] \rightarrow(0,1]$ be a $C^{3}$ piecewise differentiable map. We will say that $g$ is a Markov expanding map if $g\left(\omega_{i}\right)=$ $(0,1]$ and the restriction $\left.g\right|_{\omega_{i}}$ is a $C^{3}$ diffeomorphism with a $C^{3}$ extension to the closure for any $i \in S,\left|g^{\prime}(\theta)\right| \geq d \geq 16$ for all $\theta \in(0,1]$, and there exists $K>0$ so that $\left|g^{\prime \prime}\right| \leq K\left|g^{\prime}\right|^{2}$. The later is the so called Rènyi condition, which is a sufficient condition to obtain the bounded distortion property in Subsection 3.1. Throughout we assume that $\log \left|g^{\prime}\right| \in L^{1}$ (Leb). This is a natural assumption to obtain finite positive Lyapunov exponent for $g$ and related with the size of smaller intervals of $\mathcal{P}$. Now we introduce the family of skew-products of the space $(0,1] \times \mathbb{R}$ with countably many inverse branches.

Definition 2.1. We say that a piecewise $C^{3}$ map $\varphi:(0,1] \times \mathbb{R} \rightarrow(0,1] \times \mathbb{R}$ is a generalized Viana map if it is a skew-product given by

$$
\begin{array}{rlc}
\varphi_{\alpha}:(0,1] \times \mathbb{R} & \rightarrow & (0,1] \times \mathbb{R} \\
(\theta, x) & \mapsto\left(g(\theta), f_{\alpha}(\theta, x)\right)
\end{array}
$$

where $g$ is a piecewise linear Markov expanding map on $(0,1]$ and $f_{\alpha}(\theta, x)=$ $a_{0}+\alpha \sin (2 \pi \theta)-x^{2}$ for some $\alpha>0$ and parameter $a_{0} \in(0,2]$.

The assumptions on the parameter $a_{0}$ will be crucial. Recall that the quadratic map $h(x)=a_{0}-x^{2}$ is Misiurewicz provided that the critical point is pre-periodic repelling. It is not hard to check that there exists an interval $I_{0} \subset\left[h^{2}(0), h(0)\right]$ such that $\varphi_{\alpha}\left((0,1] \times I_{0}\right) \subset(0,1] \times I_{0}$ for every $\alpha>0$ small enough. Then we define the attractor $\Lambda=\Lambda\left(\varphi_{\alpha}\right)$ for $\varphi_{\alpha}$ by

$$
\Lambda\left(\varphi_{\alpha}\right)=\bigcap_{n \geq 0} \varphi_{\alpha}^{n}\left((0,1] \times I_{0}\right)
$$

and consider the restriction $\left.\varphi_{\alpha}\right|_{\Lambda}$. Let us mention that although it seems reasonable that some other classes of infinitely branched interval expanding maps can be considered as base dynamics without the Rènyi assumption some condition on the decay of the size of the partition elements should be necessary (e.g. otherwise could exist SRB measures without finite positive Lyapunov exponent).

Observe that we defined generalized Viana maps as a class of skewproducts. We shall consider in this space the topology that we now describe. Assume, without loss of generality, that $S=\mathbb{N}_{0}$. Given $\varepsilon>0$ we say that $\varphi:(0,1] \times \mathbb{R} \rightarrow(0,1] \times \mathbb{R}$ is $\varepsilon-C^{3}$-close to the skew-product $\varphi_{\alpha}$ above if $\varphi(\theta, x)=(\tilde{g}(\theta), \tilde{f}(\theta, x))$ is a piecewise $C^{3}$ map and satisfies: 
(i) $\varphi\left((0,1] \times I_{0}\right) \subset(0,1] \times I_{0}$, the map $\tilde{g}$ is a Markov expanding map on $(0,1]$

(ii) $(0,1]=\bigcup_{i \in S} \tilde{\omega}_{i}$ and $\left.\tilde{g}_{i}\right|_{\tilde{\omega}_{i}}: \tilde{\omega}_{i} \rightarrow(0,1]$ is a diffeomorphism that admits a $C^{3}$ extension to the boundary and is a Markov partition for $\tilde{g}$

(iii) the renormalized maps $R_{i} g: \omega_{i} \times \mathbb{R} \rightarrow(0,1]$ and $R_{i} f: \omega_{i} \times \mathbb{R} \rightarrow \mathbb{R}$ given respectively by

$$
R_{i} g(\theta)=\tilde{g}\left(\tilde{\theta}_{i+1}+\frac{\left|\tilde{\omega}_{i}\right|}{\left|\tilde{\omega}_{i}\right|}\left(\theta-\theta_{i+1}\right)\right) \quad \text { and } \quad R_{i} f(\theta, x)=\tilde{f}\left(\tilde{\theta}_{i+1}+\frac{\left|\tilde{\omega}_{i}\right|}{\left|\tilde{\omega}_{i}\right|}\left(\theta-\theta_{i+1}\right), x\right)
$$

satisfy $\sup _{x}\left\|g(\cdot)-R_{i} g(\cdot, x)\right\|_{C^{3}}<\varepsilon$ and $\left\|\left.f\right|_{\omega_{i} \times \mathbb{R}}-R_{i} f\right\|_{C^{3}}<\varepsilon$, where $|\cdot|$ denotes the Lebesgue measure of the interval $\omega_{i}$ with boundary points $\theta_{i}$ and $\theta_{i+1}$.

Let us make some comments on our assumptions. We will assume for notational simplicity that the partition $\mathcal{P}$ is preserved under perturbations, in which case $C^{3}$ perturbation coincides with the usual notion for interval maps. Condition (i) implies that the perturbed map $\varphi:(0,1] \times \mathbb{R} \rightarrow(0,1] \times$ $\mathbb{R}$ is a skew-product with countably many domains of invertibility over a Markov expanding map. These are natural assumptions if one assumes the base dynamics to be induced map from some one-dimensional nonuniformly expanding map. Condition (ii) implies the domains of invertibility of $\varphi$ to be close to those of $\varphi_{\alpha}$ and that the dynamics in each domain is $C^{3}$-close to the original one. So, these assumptions require the map $\varphi$ to be close to $\varphi_{\alpha}$ from both the topological and the differentiable viewpoints. Clearly, this class of maps include the skew-products considered in Vi97.

2.2. Statement of results. We are now in a position to state our main results.

Theorem A. Consider the skew-product $\varphi_{\alpha}:(0,1] \times I \rightarrow(0,1] \times I$ given by $\varphi_{\alpha}(\theta, x)=\left(g(\theta), f_{\alpha}(\theta, x)\right)$ and such that $h(x)=a_{0}-x^{2}$ is Misiurewicz. Then there exists $c>0$ such that for any small $\alpha$ it holds

$$
\liminf _{n \rightarrow \infty} \frac{1}{n} \log \left\|D \varphi_{\alpha}^{n}(\theta, x) v\right\| \geq c>0
$$

for Lebesgue almost every $(\theta, x)$ and every $v \in \mathbb{R}^{2} \backslash\{0\}$. Moreover, there exists $\varepsilon>0$ such that the same property holds for every $\varphi$ that is $\varepsilon-C^{3}$-close to $\varphi_{\alpha}$.

As a byproduct of the proof we obtain some estimates on the decay of the first time at which some hyperbolicity is obtained. These are known as hyperbolic times (see Al00 for some details). In consequence, one can use the works of Araújo, Solano ArS11 or Pinheiro Pi11 to build an inducing scheme and deduce the existence of an SRB measure with good statistical properties. Recall that a $\varphi$-invariant and ergodic probability measure $\mu$ is an $S R B$ measure if its basin of attraction

$$
B(\mu)=\left\{(\theta, x) \in(0,1] \times I_{0}: \frac{1}{n} \sum_{j=0}^{n-1} \delta_{\varphi^{j}(\theta, x)} \stackrel{w^{*}}{\longrightarrow} \mu\right\}
$$

has positive Lebesgue measure. Here we establish not only uniqueness of the SRB measure as we obtain several important statistical properties. Let 
$\mathcal{H}_{\beta}$ be denote the space of $\beta$-Hölder continuous observables. We obtain the following:

Theorem B. Let $\varphi_{\alpha}:(0,1] \times I \rightarrow(0,1] \times I$ be a generalized Viana map $\varphi_{\alpha}(\theta, x)=\left(g(\theta), f_{\alpha}(\theta, x)\right)$ such that $h(x)=a_{0}-x^{2}$ is Misiurewicz. Then, for any small $\alpha>0$ :

(1) $\varphi_{\alpha}$ is topologically mixing;

(2) There exists a unique $\varphi_{\alpha}$-invariant measure $\mu_{\alpha}$ that is absolutely continuous with respect to Lebesgue on the attractor $\Lambda\left(\varphi_{\alpha}\right)$;

(3) $\mu_{\alpha}$ has stretched-exponential decay of correlations, that is, there exists $C>0$ and $\tau \in(0,1)$ such that

$$
\left|\int\left(h_{1} \circ \varphi_{\alpha}^{n}\right) h_{2} d \mu_{\alpha}-\int h_{1} d \mu_{\alpha} \cdot \int h_{2} d \mu_{\alpha}\right| \leq C e^{-\tau \sqrt{n}}\left\|h_{1}\right\|_{\infty}\left\|h_{2}\right\|_{\beta}
$$

for all large $n$ and observables $h_{1} \in L^{\infty}(\mu)$ and $h_{2} \in \mathcal{H}_{\beta}$;

(4) $\mu_{\alpha}$ has stretched-exponential large deviations, meaning that there exists $\zeta \in\left(0, \frac{1}{2}\right)$ such that for all $\delta>0$ and $h \in \mathcal{H}_{\beta}$ there exists $\gamma>0$ satisfying

$$
\mu\left(\left|\frac{1}{n} \sum_{j=0}^{n-1} h \circ \varphi^{j}-\int h d \mu_{\alpha}\right|>\delta\right) \leq e^{-\gamma n^{\zeta}} \text { for all large } n ;
$$

(5) $\mu_{\alpha}$ satisfies the central limit theorem, the almost sure invariance principle, the local limit theorem and the Berry-Esseen theorem for Hölder observables

Furthermore, all these properties hold for every $\varphi$ that is $C^{3}$-close enough to $\varphi_{\alpha}$.

Our strategy to deduce the later ergodic properties is to use recent contributions to the study of stretched-exponential large deviations and limit theorems using Markov induced maps e.g. by Melbourne and Nicol [MN08, Alves, Luzzatto, Freitas, Vaienti ALFV11] or Alves and Schnelmann [AS13. Our next main result concerns the coexistence of a dense set of points with a negative and a positive Lyapunov exponents together with a full Lebesgue measure set of points with only positive Lyapunov exponents.

Theorem C. Let $\varphi_{\alpha}:(0,1] \times I \rightarrow(0,1] \times I$ be a generalized Viana map $\varphi_{\alpha}(\theta, x)=\left(g(\theta), f_{\alpha}(\theta, x)\right)$ such that $h(x)=a_{0}-x^{2}$ is Misiurewicz and let $\mathcal{V}$ be a $C^{3}$-open set of generalized Viana maps. Then there exists an open and dense subset $\mathcal{A} \subset \mathcal{V}$ such that for every $\varphi \in \mathcal{A}$

(1) there is a dense set of points $D \subset \Lambda(\varphi)$ with a negative Lyapunov exponent, that is,

$$
\limsup _{n \rightarrow \infty} \frac{1}{n} \log \left\|D \varphi^{n}(\theta, x) \frac{\partial}{\partial x}\right\|<0 \quad \text { for all }(\theta, x) \in D ;
$$

(2) Lebesgue almost every point in $\Lambda(\varphi)$ has two positive Lyapunov exponents.

In view of the previous theorem an interesting question is to understand if, at least generically, all Lyapunov exponents are bounded away from zero. The later goes in the direction of understanding possible phase transitions for 
the topological pressure $P(t)$ of the generalized Viana-map $\varphi_{\alpha}$ with respect to the family of potentials $\psi_{\alpha, t}=-t \log \left|\partial_{x} \varphi_{\alpha}\right|$, that is, parameters $t$ such that $\psi_{\alpha, t}$ has none or more than one equilibrium state. For that purpose it would be important to characterize the range of the entropy among invariant and ergodic measures with one negative Lyapunov exponent.

This final part of the section is devoted to a better understanding of the dynamics of quadratic skew-products where the parameters are driven among hyperbolic parameters. In general, to obtain hiperbolicity for the composition of fibered polynomials is a hard question. Jonsson [Jo97 and Sester [Se99] studied this topic in the complex variable setting and, in particular, obtained conditions equivalent to hyperbolicity. With this in mind we state our last main result. For simplicity, we will say that an ergodic measure is hyperbolic if is has only nonzero Lyapunov exponents and not all of the same sign.

Theorem D. Let I denote a closed interval. Assume that $X$ is a compact Riemannian manifold, that a continuous map $S: X \rightarrow X$ admits a unique $S R B$ measure $\mu_{S}$, that $T: I \rightarrow I$ is a hyperbolic polynomial with negative Schwarzian derivative and $a: X \rightarrow \mathbb{R}$ is a $C^{3}$-smooth function. There exists $\varepsilon>0$ such that if $\|a\|_{C^{3}}<\varepsilon$ then the skew product

$$
\psi:(x, y) \mapsto(S(x), T(y)+a(x))
$$

is such that $\psi$ has an ergodic SRB measure $\nu$ whose basin of attraction contains Lebesgue almost every point in some open and proper subset of $X \times I$. If, in addition, $\mu_{S}$ is hyperbolic then $\nu$ is also hyperbolic.

Let us mention that it is not clear to us wether the transversality condition of admissible curves can be used to prove that the hyperbolic SRB measure is absolutely continuous with respect to Lebesgue. Related results were obtained by Tsujii Tsu01 and Volk Vo11. Finally, the previous result applies for different coupling functions $S$ as rotations, $C^{2}$-Maneville-Pommeau maps and, in particular, directly for Viana maps whose parameters are driven along hyperbolic one as follows.

Corollary A. Consider the skew-product $\varphi_{\alpha}: S^{1} \times I \rightarrow S^{1} \times I$ given by $\varphi_{\alpha}(\theta, x)=\left(g(\theta), f_{\alpha}(\theta, x)\right)$, where $g(\theta)=d \theta(\bmod 1)$ and $f_{\alpha}(\theta, x)=a_{0}+$ $\alpha \sin (2 \pi \theta)-x^{1}$ for $a_{0}$ so that $h(x)=a_{0}-x^{2}$ is hyperbolic. Then for every small $\alpha>0$ the map $\varphi_{\alpha}$ has an $S R B$ measure $\nu$, it is hyperbolic and supported in a proper attractor. Moreover, the same property holds for every $\varphi$ that is $C^{3}$-close enough to $\varphi_{\alpha}$.

Let us mention that the SRB measure above is supported on a topological atractor $\mathcal{G} \subset(0,1] \times I_{0}$ as described in detail in Section 7 . Finally, some interesting questions are to understand if the complement $K$ of the attractor $\mathcal{G}$ is such that the intersection $K_{\theta}$ with the fiber over $\theta$ is a totally disconnected, compact set of zero Lebesgue measure. For that it would be necessary to prove that $K$ is an expanding set for $\varphi_{\alpha}$. Moreover, since almost every parameter is regular or stochastic for the quadratic map it would be interesting to understand wether either of Theorem A or Corollary A hold for quadratic skew-products and Lebesgue almost every parameter $a \in(0,2]$. 
2.3. Some applications. Let us finish this section with some examples.

Example 2.2 (Viana maps). The class of maps considered in Vi97 fit in the previous setting. In fact, assume $d \geq 16$ and take the Markov expanding map on $(0,1]$ given by $g(\theta)=d \theta-[d \theta]$ (where [.] stands for the integer part). Then $g$ admits a $C^{3}$-extension to the boundary elements and one can identify the boundary points of $(0,1]$ to obtain the $C^{3}$ expanding map on the circle $\mathbb{S}^{1}$ given by $g(\theta)=d \theta(\bmod 1)$, thus recovering the previous setting. In particular, in this context Theorems $\mathrm{A}$ and $\mathrm{B}$ are consequences of [Vi97, Al00].

In this context, it follows from Theorems $\mathrm{C}$ that $C^{1}$-generic transformations in the $C^{3}$ neighborhood of Viana maps exhibit coexistence of a dense set of points with one negative Lyapunov exponent while Lebesgue almost every point has only positive Lyapunov exponents. Finally, it follows from Theorem $\mathrm{A}$ that quadratic skew-products with parameters driven among hyperbolic ones admit a unique SRB measure, it is hyperbolic and the complement of its basin of attraction is an expanding Cantor set of lines.

In the next class of examples we present a robust class of Markov expanding maps with discontinuities and infinitely many invertibility domains.

Example 2.3 (Quadratic skew-products over piecewise linear expanding maps). Let $\mathcal{P}$ be an arbitrary countable partition of the unit interval $(0,1]$ in subintervals $\left(\omega_{i}\right)_{i \in S}$ with size smaller or equal to $\frac{1}{d}$ and let $g_{0}$ be piecewise linear satisfying $g\left(\omega_{i}\right)=(0,1]$. Since $\left|g_{0}^{\prime}(\theta)\right| \geq d$ and $g_{0}^{\prime}(\theta)^{\prime}=0$ for all $\theta \in(0,1]$ then it is clear that $g_{0}$ is a piecewise Markov expanding map and satisfies the Rènyi condition. See Figure 1.

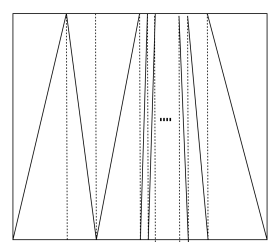

$g_{0}$

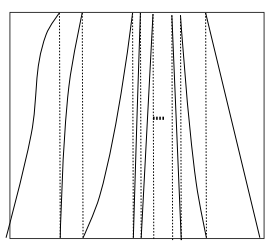

$g$

FigURE 1. Small perturbation of piecewise linear expanding map

Moreover, if $\tilde{g}$ is a Markov expanding map that is $\varepsilon$ - $C^{3}$-close enough to $g$ then it follows from Lemma 3.1 that it also satisfies the Rènyi condition thus satisfying all the hypothesis to be used as base dynamics.

\section{Preliminaries}

In this section we recall some definitions and preliminaries that will be used in the proof of the main results.

\subsection{One dimensional dynamics.}


Combinatorial description of Markov expanding maps. Here we describe the Markov expanding maps $g$ from the combinatorial point of view. Let $\mathcal{P}=$ $\left\{\omega_{i}\right\}_{i \in S, S \subset \mathbb{N}}$ be the Markov partition for $g$. Then there is a semi-conjugacy between the dynamics of $g$ and the full shift $\sigma: S^{\mathbb{N}} \rightarrow S^{\mathbb{N}}$ given by

$$
\sigma\left(s_{0}, s_{1}, s_{2}, \ldots\right)=\left(s_{1}, s_{2}, s_{3}, \ldots\right),
$$

where the semi-conjugation is given by the itinerary map $\iota: \mathbb{N}^{\mathbb{N}} \rightarrow(0,1]$ defined as $\iota\left(s_{0}, s_{1}, s_{2}, \ldots\right)=\theta$ and $\theta$ is the only point in $(0,1]$ satisfying $g^{j}(\theta) \in \omega_{s_{j}}$ for all $j$. Set $\mathcal{P}^{(n)}=\bigvee_{j=0}^{n-1} g^{-j}(\mathcal{P})$ and for any partition element $\omega \in \mathcal{P}^{(n)}$ define $\iota_{n}=\iota_{n}(\omega)=\left(s_{1}, s_{2}, \ldots, s_{n-1}\right)$ as its $n$-th itinerary. For simplicity we will denote by $\omega_{\left(s_{0}, s_{1}, s_{2}, \ldots, s_{n-1}\right)}$ the element of $\mathcal{P}^{(n)}$ whose itinerary is $\left(s_{0}, s_{1}, s_{2}, \ldots, s_{n-1}\right)$. This will be helpful to give a precise description of points that visit to definite regions of the phase space.

Rènyi condition. Here we show that Rényi condition is an open property among Markov expanding maps and relate this with the bounded distortion property.

Lemma 3.1. Assume that $g:(0,1] \rightarrow(0,1]$ is a $C^{3}$-Markov expanding map satisfying the Rènyi condition $\left|g^{\prime \prime}\right| \leq K\left|g^{\prime}\right|^{2}$. If $\|g-\tilde{g}\|_{C^{3}}<\varepsilon$ for small $\varepsilon$ then $\tilde{g}$ satisfies $\left|\tilde{g}^{\prime \prime}\right| \leq \tilde{K}\left|\tilde{g}^{\prime}\right|^{2}$ with $\tilde{K}=(d-\varepsilon)^{-2} \varepsilon+(1-\varepsilon)^{-2} K$.

Proof. Assume that $\|g-\tilde{g}\|_{C^{3}}<\varepsilon$. Using that $g$ is expanding it follows that $\left|g^{\prime}-\tilde{g}^{\prime}\right|<\varepsilon<\varepsilon\left|g^{\prime}\right|$ and consequently

$$
\begin{aligned}
\frac{\left|\tilde{g}^{\prime \prime}\right|}{\left|\tilde{g}^{\prime}\right|^{2}} & \leq \frac{\left|g^{\prime \prime}\right|+\left|g^{\prime \prime}-\tilde{g}^{\prime \prime}\right|}{\left|\tilde{g}^{\prime}\right|^{2}} \leq \frac{1}{(d-\varepsilon)^{2}}\|g-\tilde{g}\|_{C^{2}}+\frac{\left|g^{\prime \prime}\right|}{\left(\left|g^{\prime}\right|-\left|g^{\prime}-\tilde{g}^{\prime}\right|\right)^{2}} \\
& \leq \frac{1}{(d-\varepsilon)^{2}}\|g-\tilde{g}\|_{C^{2}}+\frac{1}{(1-\varepsilon)^{2}} \frac{\left|g^{\prime \prime}\right|}{\left|g^{\prime}\right|^{2}} \leq \frac{1}{(d-\varepsilon)^{2}}\|g-\tilde{g}\|_{C^{2}}+\frac{K}{(1-\varepsilon)^{2}} .
\end{aligned}
$$

This proves that $\tilde{g}$ also satisfies the Rènyi condition and proves the lemma.

In the second lemma we collect some bounded distortion estimates.

Lemma 3.2. Let $g$ be a $C^{3}$-Markov expanding map satisfying $\left|g^{\prime}(\theta)\right| \geq d$ and the Rènyi condition $\left|g^{\prime \prime}\right| \leq K\left|g^{\prime}\right|^{2}$. Then, for all $\omega \in \mathcal{P}^{(n)}$ and $\theta_{1}, \theta_{2} \in \omega$

$$
\exp \left(-\frac{d K}{d-1}\right) \leq \frac{\left|\left(g^{n}\right)^{\prime}\left(\theta_{1}\right)\right|}{\left|\left(g^{n}\right)^{\prime}\left(\theta_{2}\right)\right|} \leq \exp \left(\frac{d K}{d-1}\right) .
$$

Proof. Let $\theta_{1}, \theta_{2} \in \omega$ for some $\omega \in \mathcal{P}^{(n)}$ be given. We may assume, without loss of generality, that $\left.g^{n}\right|_{\omega}$ is increasing. Then

$$
\begin{aligned}
\left|\log \frac{\left(g^{n}\right)^{\prime}\left(\theta_{1}\right)}{\left(g^{n}\right)^{\prime}\left(\theta_{2}\right)}\right| & \leq \sum_{j=0}^{n-1}\left|\log \left(g^{\prime}\left(g^{j}\left(\theta_{1}\right)\right)\right)-\log \left(g^{\prime}\left(g^{j}\left(\theta_{2}\right)\right)\right)\right| \\
& =\sum_{j=0}^{n-1}\left|\int_{g^{j}\left(\theta_{1}\right)}^{g^{j}\left(\theta_{2}\right)} \frac{g^{\prime \prime}(\theta)}{g^{\prime}(\theta)} d \theta\right| \leq K \sum_{j=0}^{n-1}\left|\int_{g^{j}\left(\theta_{1}\right)}^{g^{j}\left(\theta_{2}\right)} g^{\prime}(\theta) d \theta\right| \\
& =K \sum_{j=1}^{n}\left|g^{j}\left(\theta_{1}\right)-g^{j}\left(\theta_{2}\right)\right| \leq K \sum_{j=1}^{n} d^{-(n-j)}\left|g^{n}\left(\theta_{1}\right)-g^{n}\left(\theta_{2}\right)\right|
\end{aligned}
$$


which is clearly bounded from above by $K\left(1-d^{-1}\right)^{-1}$. Since $\theta_{1}, \theta_{2}$ were arbitrary then the lower bound also holds. This finishes the proof of the lemma.

Finally let us recall that it is well known that if $g$ is a Markov expanding map with the Rènyi condition then there exists a unique absolutely continuous invariant probability measure. We will use the following strong Gibbs property for Lebesgue.

Corollary 3.3. Let $g$ be a $C^{3}$-Markov expanding map satisfying $\left|g^{\prime}(\theta)\right| \geq d$ and the Rènyi condition $\left|g^{\prime \prime}\right| \leq K\left|g^{\prime}\right|^{2}$. Then, for all $\omega \in \mathcal{P}^{(n)}$ and $\theta^{*} \in \omega$

$$
\exp \left(-\frac{d K}{d-1}\right) \leq \frac{\operatorname{Leb}(\omega)}{\left|\left(g^{n}\right)^{\prime}\left(\theta^{*}\right)\right|^{-1}} \leq \exp \left(\frac{d K}{d-1}\right)
$$

Proof. The proof is a simple application of the usual change of coordinates to the diffeomorphism $\left.g^{n}\right|_{\omega}: \omega \rightarrow(0,1]$ by means that

$$
\int_{\omega}\left|\left(g^{n}\right)^{\prime}(\theta)\right| d \theta=\operatorname{Leb}\left(g^{n}(\omega)\right)=\operatorname{Leb}(f(0,1])=1
$$

together with the previous bounded distortion estimates.

Hyperbolicity in dimension one. In [Sm00], Smale proposed the density of hyperbolicity in dimension one as one of the problems for the 21st century. Recall that a $C^{1}$ endomorphism of a compact interval is hyperbolic if it has finitely many hyperbolic attracting periodic points and the complement of the basins of attraction is a hyperbolic set. Such major achievement was obtained by Kozlovski, Shen and van Strien.

Theorem 3.4. (Theorem 2 in KSvS07a]) Hyperbolic maps are dense in the space of $C^{k}$ maps of the compact interval or the circle for $k=1,2, \ldots, \infty, \omega$.

Fibered expansion. Now we collect some results on a mechanism to obtain expansion in the fiber direction. Roughly, expansion for random composition of perturbations of a Misiurewicz quadratic map $h(x)=a_{0}-x^{2}$ is obtained if orbits avoid the critical region and the loss of expansion in each return to the critical region is proportional to the return depth. More precisely,

Proposition 3.5. Vi97, Lemmas 2.4 and 2.5] Given $\alpha>0$, take $h(x)=$ $a_{0}-x^{2}, f(\theta, x)=a_{0}+\alpha \sin (2 \pi \theta)-x^{2}$ and $\varphi(\theta, x)=\left(g(\theta), f_{\alpha}(\theta, x)\right)$. There are constants $0<\kappa<1$ and $0<\eta \leq \frac{1}{3}$ (depending only on $h$ ) and $\delta_{1}, C_{2}>0$, $\sigma_{1}, \sigma_{2}>1$ such that, for every small $\alpha>0$ there exists $N(\alpha) \geq 1$ satisfying:

(1) $K_{0} \log \frac{1}{\alpha} \leq N(\alpha) \leq K_{1} \log \frac{1}{\alpha}$ for some uniform constants $K_{0}, K_{1}>$ 0 ;

(2) Given an interval $I \subset I_{0}$, for every $(\theta, x) \in(0,1] \times I$ with $|x|<$ $2 \sqrt{\alpha}$ the iterates $\left(\theta_{j}, x_{j}\right)=\varphi^{j}(\theta, x)$ satisfy $\left|x_{j}\right| \geq \sqrt{\alpha}$ for every $j=$ $1 \ldots N(\alpha)$;

(3) $\prod_{j=0}^{N(\alpha)-1}\left|\partial_{x} f\left(\theta_{j}, x_{j}\right)\right| \geq|x| \alpha^{-1+\eta}$ for all $(\theta, x) \in(0,1] \times I$ with $|x|<$ $2 \sqrt{\alpha}$

(4) For every $(\theta, x) \in(0,1] \times I$ with $\sqrt{\alpha} \leq|x|<\delta_{1}$ there exists $p(x) \leq$ $N(\alpha)$ so that $\prod_{j=0}^{p(x)-1}\left|\partial_{x} f\left(\theta_{j}, x_{j}\right)\right| \geq \frac{1}{\kappa} \sigma_{1}^{p(x)}$; 
(5) $\prod_{j=0}^{n-1}\left|\partial_{x} f\left(\theta_{j}, x_{j}\right)\right| \geq C_{2} \sqrt{\alpha} \sigma_{2}^{n}$ for every $(\theta, x) \in(0,1] \times I$ with $\left|x_{j}\right| \geq$ $\sqrt{\alpha}$ for every $j=1, \ldots, n-1$; and

(6) $\prod_{j=0}^{n-1}\left|\partial_{x} f\left(\theta_{j}, x_{j}\right)\right| \geq C_{2} \sigma_{2}^{n}$ for all $(\theta, x) \in(0,1] \times I$ such that $\left|x_{j}\right| \geq$ $\sqrt{\alpha}$ with $j=1, \ldots, n-1$ and $\left|x_{n}\right| \leq \delta_{1}$.

Taking the previous proposition into account it is important to estimate how close typical points return close to the critical region.

3.2. Partial hyperbolicity and admissible curves. Under our assumptions on the generalized Viana maps of Definition 2.1, we obtain that the map is indeed partially hyperbolic in the sense that the dynamics along the horizontal direction dominates the dynamics along the vertical fibers. This will be made precise in terms of admissible curves as we now describe.

Definition 3.6. A curve $\hat{Y}=\operatorname{graph}(Y)$ with $Y:(0,1] \rightarrow I_{0}$ is an admissible curve if it is $C^{2}$ differentiable, $\left|Y^{\prime}(\theta)\right| \leq \alpha$ and $\left|Y^{\prime \prime}(\theta)\right| \leq \alpha$ for every $\theta \in$ $(0,1]$.

The strong expansion assumption on the Markov map $g$ yields a domination property as we now describe.

Lemma 3.7. If $\hat{Y}$ is an admissible curve then for every $\omega \in \mathcal{P}^{(n)}$ it follows that $\varphi^{n}\left(\left.\hat{Y}\right|_{\omega}\right)$ is an admissible curve. In particular $\varphi^{n}(\hat{Y})$ is an at most countable collection of admissible curves.

Proof. This lemma follows from [Vi97, Lemma 2.1], whose argument we reproduce here for completeness and the reader's convenience. Since it is enough to prove the lemma for $n=1$ and use the argument recursively, let $\hat{Y}=\operatorname{graph} Y$ be an admissible curve and take $\omega \in \mathcal{P}$. Then, for every $\theta \in P$

$$
\varphi(\hat{Y}(\theta))=\varphi(\theta, Y(\theta))=(g(\theta), f(\theta, Y(\theta)))=\left(g(\theta), Y_{1}(g(\theta))\right)
$$

where, by the chain rule and definition of $Y_{1}:(0,1] \rightarrow I$,

$$
\left|Y_{1}^{\prime}(g(\theta))\right| \leq \frac{1}{\left|g^{\prime}(\theta)\right|}\left|\partial_{\theta} f(\hat{Y}(\theta))+\partial_{x} f(\hat{Y}(\theta)) Y^{\prime}(\theta)\right| \leq \frac{2 \pi+4}{16} \alpha<\alpha .
$$

Analogously it is not hard to check that

$$
\begin{aligned}
\left|Y_{1}^{\prime \prime}(g(\theta))\right| \leq \frac{1}{\left|g^{\prime}(\theta)\right|^{2}} & \mid \partial_{\theta \theta} f(\hat{Y}(\theta))+\partial_{x \theta} f(\hat{Y}(\theta)) Y^{\prime}(\theta)+\partial_{\theta x} f(\hat{Y}(\theta)) Y^{\prime}(\theta) \\
& +\partial_{x} f(\hat{Y}(\theta)) Y^{\prime \prime}(\theta)+\partial_{x x} f(\hat{Y}(\theta))\left(Y^{\prime}(\theta)\right)^{2}-Y_{1}^{\prime}(g(\theta)) g^{\prime \prime}(\theta) \mid,
\end{aligned}
$$

which is smaller than $\alpha$ since the partial derivatives of $f$ are smaller compared with the term $1 /\left|g^{\prime}\right|^{2} \leq 1 / 16^{2}$. This proves that $\hat{Y}_{1}=\varphi\left(\left.\hat{Y}\right|_{\omega}\right)$ is an admissible curve. Since $\mathcal{P}$ is at most countable then $\varphi(\hat{Y})$ is the union of at most countable admissible curves. This finishes the proof of the lemma.

The crucial property of admissible curves is that their images by $\varphi$ are non-flat.

Lemma 3.8. Let $\hat{Y}=$ graph $Y$ be an admissible curve and set $\hat{Y}_{1}(\theta)=$ $\varphi(\hat{Y}(\theta))=\left(g(\theta), Y_{1}(\theta)\right)$. Then $\left|Y_{1}^{\prime}(\theta)\right| \geq \alpha / 2$ or $\left|Y_{1}^{\prime \prime}(\theta)\right| \geq 4 \alpha$ for every $\theta \in(0,1]$ and

$$
\operatorname{Leb}\left(\theta \in(0,1]: \hat{Y}_{1}(\theta) \in(0,1] \times I\right) \leq \frac{6|I|}{\alpha}+2 \sqrt{\frac{|I|}{\alpha}} .
$$


for any interval $I \subset I_{0}$.

Proof. The proof follows the same ideas of [Vi97, Lemma 2.2] even with the presence of discontinuities for $g$. In fact, set $\hat{Y}(\theta)=(\theta, Y(\theta))$ and notice that for all $\theta \in(0,1]$

$$
Y_{1}^{\prime}(\theta)=\partial_{\theta} f(\hat{Y}(\theta))+\partial_{x} f(\hat{Y}(\theta)) Y^{\prime}(\theta)=2 \pi \alpha \cos (2 \pi \theta)-2 Y(\theta) Y^{\prime}(\theta)
$$

and $Y_{1}^{\prime \prime}(\theta)=-4 \pi^{2} \sin (2 \pi \theta)-2 Y^{\prime}(\theta)^{2}-2 Y(\theta) Y^{\prime \prime}(\theta)$. If $\theta \in \mathcal{A}=\{\tilde{\theta} \in$ $\left.(0,1]:|\sin (2 \pi \tilde{\theta})| \leq \frac{1}{3}\right\}$ then $|\cos (2 \pi \theta)| \geq \frac{11}{12}$ and it follows from (3.1) that $\left|Y_{1}^{\prime}(\theta)\right| \geq\left(\frac{11 \pi}{6}-4\right) \alpha>\frac{\alpha}{2}$. Otherwise, for $\theta \in(0,1] \backslash \mathcal{A}$ it follows that $\left|Y_{1}^{\prime \prime}(\theta)\right| \geq\left(\frac{4 \pi^{2}}{3}-2-2 \alpha\right) \alpha \geq 4 \alpha$. This proves the first statement of the lemma.

Now, notice that $\mathcal{A}$ has three connected components and $\left|Y_{1}^{\prime}(\theta)\right| \geq \alpha / 2$ for all $\theta \in \mathcal{A}$. Moreover, since the map $\theta \mapsto Y_{1}(\theta)$ is $C^{1}$-differentiable then applying the Mean Value Theorem applied to $Y_{1}$ on each connected component of $\mathcal{A}$ it follows that $\operatorname{Leb}\left(\theta \in \mathcal{A}: \hat{Y}_{1}(\theta) \in(0,1] \times I\right) \leq \frac{6|I|}{\alpha}$. Using that $\left|Y_{1}^{\prime \prime}\right| \geq 4 \alpha$ on the two connected components of $(0,1] \backslash \mathcal{A}$ a similar argument shows that $\operatorname{Leb}\left(\theta \in(0,1] \backslash \mathcal{A}: \hat{Y}_{1}(\theta) \in(0,1] \times I\right) \leq 4 \sqrt{\frac{|I|}{4 \alpha}}=2 \sqrt{\frac{|I|}{\alpha}}$. The proof of the lemma is now complete.

We obtain the following very useful consequence.

Corollary 3.9. Let $\hat{Y}=\operatorname{graph}(Y)$ be an admissible curve and set $\hat{Y}_{j}=$ $\varphi^{j}(\hat{Y})$. Then there exists $C>0$ (depending only on $g$ ) such that for any subinterval satisfying $|I| \leq \alpha$ it holds

$$
\operatorname{Leb}\left(\theta \in(0,1]: \hat{Y}_{j}(\theta) \in(0,1] \times I\right) \leq C \sqrt{\frac{|I|}{\alpha}} \quad \text { for all } j \geq 1 \text {. }
$$

Proof. First note that the case $j=1$ corresponds to the previous lemma. Hence, let $j \geq 2$ be arbitrary and fixed. If $\omega \in \mathcal{P}^{(j-1)}$ then $g^{j-1}(\omega)=$ $(0,1]$ and $\varphi^{j-1}\left(\left.\hat{Y}\right|_{\omega}\right)$ is an admissible curve. Therefore, $\operatorname{Leb}(\theta \in(0,1]$ : $\left.\varphi\left(\varphi^{j-1}\left(\left.\hat{Y}\right|_{\omega}(\theta)\right)\right) \in(0,1] \times I\right) \leq 6 \sqrt{\frac{|I|}{\alpha}}$ for any subinterval $I$ satisfying $|I| \leq \alpha$. Then one can use the bounded distortion property to get

$$
\begin{aligned}
\operatorname{Leb}\left(\theta \in(0,1]: \hat{Y}_{j}(\theta) \in \hat{I}\right) & =\sum_{\omega \in \mathcal{P}^{(j-1)}} \operatorname{Leb}\left(\theta \in \omega: \varphi\left(\varphi^{j-1}\left(\left.\hat{Y}\right|_{\omega}(\theta)\right)\right) \in \hat{I}\right) \\
& \leq \sum_{\omega \in \mathcal{P}^{(j-1)}} 6 \frac{K d}{d-1} \sqrt{\frac{|I|}{\alpha}}|\omega| \leq C \sqrt{\frac{|I|}{\alpha}},
\end{aligned}
$$

where $\hat{I}=(0,1] \times I$ and $C=6 K>0$ depends only on $g$. The proof of the corollary is now complete.

Remark 3.10. Let us mention that the bound in the right hand side of the expression in Corollary 3.9 depends on $\alpha$ and it increases when $\alpha$ approaches zero. In fact, as $\alpha$ will be required to be very small for the large deviations argument in Subsection 4.2, it is necessary to obtain similar estimates where the right hand side above does not depend on $\alpha$. 


\section{Positive Lyapunov exponents For the SkeW-Product $\varphi_{\alpha}$}

In this section we study the recurrence of typical points near the critical region and deduce existence of positive Lyapunov exponents Lebesgue almost everywhere.

4.1. Recurrence estimates. For notational simplicity set $\hat{J}(r)=(0,1] \times$ $J(r)$ for every $r$, with $J(r)=\left[-\sqrt{\alpha} e^{-r}, \sqrt{\alpha} e^{-r}\right]$. In the next proposition we show that for a sufficiently large iterate $\varphi^{M(\alpha)}$ of $\varphi$ the measure of the set of points which have exponential deep returns decay exponentially fast with a rate that does not involve $\alpha$. More precisely, if $0<\eta<\frac{1}{3}$ is as given in Propositon 3.5 we obtain the following.

Proposition 4.1. There exists $\varepsilon>0$ small so that every $\varphi$ that is $\varepsilon-C^{3}$ close to $\varphi_{\alpha}$ satisfies the following property: there exists $\tilde{C}, \beta>0$ and for any given $\alpha>0$ there is a positive integer $M=M(\alpha)<N(\alpha)$ such that if $\hat{Y}=\operatorname{graph}(Y)$ is an admissible curve, then

$$
\operatorname{Leb}\left(\theta \in(0,1]: \hat{Y}_{M}(\theta) \in \hat{J}(r-2)\right) \leq \tilde{C} e^{-5 \beta r}
$$

for every $r \geq\left(\frac{1}{2}-2 \eta\right) \log \frac{1}{\alpha}$.

Proof. Let $\hat{Y}$ be a fixed arbitrary admissible curve and set $\hat{Y}_{j}(\theta)=\varphi_{\alpha}^{j}(\hat{Y}(\theta)=$ $\left(g^{j}(\theta), Y_{j}(\theta)\right)$. On the one hand using Corollary 3.9 we deduce that

$$
\operatorname{Leb}\left(\theta \in(0,1]: \hat{Y}_{j}(\theta) \in \hat{J}(r-2)\right) \leq C \alpha^{-\frac{1}{2}} \sqrt{|J(r-2)|} \leq C \alpha^{-\frac{1}{4}} e^{-\frac{1}{2}(r-2)},
$$

which satisfies the assertion in the corollary with $\tilde{C}=C e$ and $\beta=\frac{1}{15}$, provided that $\frac{r}{6} \geq \frac{1}{4} \log \frac{1}{\alpha}$. So, through the remaining we assume $\left(\frac{1}{2}-2 \eta\right) \log \frac{1}{\alpha} \leq$ $r \leq \frac{3}{2} \log \frac{1}{\alpha}$. Let $M=M(\alpha)$ be maximal such that $32^{M} \alpha \leq 1$, and note that $M<N$. One can write

$$
\begin{aligned}
\operatorname{Leb}\left(\theta \in(0,1]: \hat{Y}_{M}(\theta) \in \hat{J}(r-2)\right) & =\sum_{\omega \in \mathcal{P}(M)} \operatorname{Leb}\left(\theta \in \omega: \hat{Y}_{M}(\theta) \in \hat{J}(r-2)\right) \\
& =\sum_{\underline{s} \in S^{M}} \operatorname{Leb}\left(\theta \in \omega_{\underline{s}}: \hat{Y}_{M}(\theta) \in \hat{J}(r-2)\right),
\end{aligned}
$$

where $\underline{s}=\left(s_{1}, s_{2}, \ldots, s_{M}\right) \in S^{M}$ is the itinerary for the elements of $\mathcal{P}^{(M)}$. The strategy is to subdivide itineraries $\underline{s}=\left(s_{1}, s_{2}, \ldots, s_{M}\right) \in S^{M}$ according to its average depth $\sum_{i=1}^{M} s_{i}(\theta)$. On the other hand, we use a large deviations argument to show that points with large average depth decrease exponentially fast. On the other hand, admissible curves associated to points with smaller average have vertical displacement.

Claim: There exists $\zeta \in(0,1)$ and for any admissible curve $\hat{Z}$ there are disjoint collections $\mathcal{P}_{1}$ and $\mathcal{P}_{2}$ of elements of $\mathcal{P}$ such that the image $\hat{Z}_{1}(\theta)=$ $\varphi(\hat{Z})(\theta)=\left(g(\theta), Z_{1}(\theta)\right)$ satisfies $\left|Z_{1}\right|_{\omega}-Z_{1}|\tilde{\omega}| \geq \frac{\alpha}{100}$ for all $\omega \in \mathcal{P}_{1}$ and $\tilde{\omega} \in \mathcal{P}_{2}$ and

$$
\zeta \leq \operatorname{Leb}\left(\bigcup_{\omega \in \mathcal{P}_{1}} \omega\right) \leq \operatorname{Leb}\left(\bigcup_{\omega \in \mathcal{P}_{2}} \omega\right) \leq 1-\zeta
$$


Proof of the Claim: It follows from Lemma 3.8 that the admissible curve $\hat{Z}_{1}$ has two critical points $\theta_{1}^{\prime}<\theta_{2}^{\prime}$ one in each connected component of the set $(0,1] \backslash \mathcal{A}=\left\{\theta \in(0,1]:|\sin (2 \pi \theta)|>\frac{1}{3}\right\}$. Hence $\frac{1}{2 \pi} \arcsin \left(\frac{1}{3}\right)<\theta_{1}^{\prime}<$ $\frac{1}{2}-\frac{1}{2 \pi} \arcsin \left(\frac{1}{3}\right)$ and also $\frac{1}{2}+\frac{1}{2 \pi} \arcsin \left(\frac{1}{3}\right)<\theta_{2}^{\prime}<1-\frac{1}{2 \pi} \arcsin \left(\frac{1}{3}\right)$.

On the one hand, if $\theta_{1}^{\prime}, \theta_{2}^{\prime} \notin\left[\frac{1}{4}, \frac{3}{4}\right]$ then $\left[\theta_{1}^{\prime}, \theta_{1}^{\prime}+\frac{1}{16}\right)$ and $\left(\theta_{2}^{\prime}-\frac{1}{16}, \theta_{2}^{\prime}\right]$ are disjoint intervals that do not intersect the middle component $\left[\frac{1}{2}-\frac{1}{2 \pi} \arcsin \left(\frac{1}{3}\right), \frac{1}{2}+\right.$ $\left.\frac{1}{2 \pi} \arcsin \left(\frac{1}{3}\right)\right]$ in $\mathcal{A}$. Then, using that $\left.\hat{Z}_{1}\right|_{\left[\theta_{1}^{\prime}, \theta_{2}^{\prime}\right]}$ is strictly monotone and $\left|Z_{1}^{\prime}\right|_{\mathcal{A}} \mid \geq \frac{\alpha}{2}$

$$
\left.\inf Z_{1}\right|_{\left[\theta_{1}^{\prime}, \theta_{1}^{\prime}+\frac{1}{16}\right]}-\left.\sup Z_{1}\right|_{\left[\theta_{2}^{\prime}-\frac{1}{16}, \theta_{2}^{\prime}\right]} \geq \frac{\alpha}{2 \pi} \arcsin \left(\frac{1}{3}\right) \geq \frac{\alpha}{100} .
$$

On the other hand, if $\theta_{1}^{\prime} \geq \frac{1}{4}$ then $\left(0, \frac{1}{16}\right]$ and $\left(\theta_{1}^{\prime}-\frac{1}{16}, \theta_{1}^{\prime}\right]$ are disjoint intervals. Moreover, using that $\left.Z_{1}\right|_{\left(0, \theta_{1}^{\prime}\right]}$ is strictly monotone and that $\left|Z_{1}^{\prime \prime}\right|_{\left(\frac{1}{16}, \frac{3}{16}\right]} \mid \geq 4 \alpha$ we obtain analogouly inf $\left.Z_{1}\right|_{\left(\theta_{1}^{\prime}-\frac{1}{16}, \theta_{1}^{\prime}\right]}-\left.\sup Z_{1}\right|_{\left(0, \frac{1}{16}\right]} \geq \frac{4 \alpha}{64} \geq$ $\frac{\alpha}{100}$. A similar reasoning holds for the case $\theta_{2}^{\prime} \leq \frac{3}{4}$.

Since all partition elements of $\mathcal{P}$ have length smaller or equal to $\frac{1}{16}$, there are collections $\mathcal{P}_{1}^{\prime}$ and $\mathcal{P}_{2}^{\prime}$ of elements in $\mathcal{P}$ such that inf $\left.Z_{1}\right|_{\omega}-\left.\sup Z_{1}\right|_{\tilde{\omega}} \geq$ $\frac{\alpha}{100}$ for all $\omega \in \mathcal{P}_{1}^{\prime}$ and $\tilde{\omega} \in \mathcal{P}_{2}^{\prime}$. In addition, using that $\mathcal{P}_{1}^{\prime}$ and $\mathcal{P}_{2}^{\prime}$ are disjoint we get

$$
1-\frac{1}{16} \geq \operatorname{Leb}\left(\bigcup_{\omega \in \mathcal{P}_{i}^{\prime}} \omega\right) \geq \frac{1}{16}
$$

for $i=1,2$ and we set $\zeta=\frac{1}{16}$. Finally if $\operatorname{Leb}\left(\bigcup_{\omega \in \mathcal{P}_{1}} \omega\right) \leq \operatorname{Leb}\left(\bigcup_{\omega \in \mathcal{P}_{2}} \omega\right)$ we define $\left(\mathcal{P}_{1}, \mathcal{P}_{2}\right):=\left(\mathcal{P}_{1}^{\prime}, \mathcal{P}_{2}^{\prime}\right)$ while otherwise $\left(\mathcal{P}_{1}, \mathcal{P}_{2}\right):=\left(\mathcal{P}_{2}^{\prime}, \mathcal{P}_{1}^{\prime}\right)$. This finishes the proof of our claim.

Let $\omega, \tilde{\omega} \in \mathcal{P}^{(M)}$ be arbitrary with $\iota_{M}(\omega)=\left(s_{1}, \ldots, s_{M}\right)$ and $\iota_{M}(\tilde{\omega})=$ $\left(\tilde{s}_{1}, \ldots, \tilde{s}_{M}\right)$, and assume that $\left|Y_{M}(\theta)\right|<\sqrt{\alpha}$ for some $\theta$ since otherwise there is nothing to prove. We collect some facts whose proof can be found in [Vi97, p.72-73]:

(1) (Expansion estimates) Given an arbitrary $\hat{y} \in \hat{Y}$ and $0 \leq j \leq M-1$

$$
\lambda_{j}:=\left|\partial_{x} f^{M-j}\left(\varphi_{\alpha}^{j}(\hat{y})\right)\right| \geq C_{2} \sigma_{2}^{M-j}
$$

(2) (Bounded distortion) For all $0 \leq j \leq M-1$, all $\left(\theta_{j}, x_{j}\right) \in \hat{Y}_{j}$ and $1 \leq i \leq M-j$ it holds that

$$
\frac{1}{2} \frac{\lambda_{j}}{\lambda_{i+j}} \leq\left|\partial_{x} f^{i}\left(\theta_{j}, x_{j}\right)\right| \leq 2 \frac{\lambda_{j}}{\lambda_{i+j}}
$$

(3) (Positive frequency) If $K=400 e^{2}, t_{1}=1$ and define recursively $t_{i+1}=\min \left\{t_{i}<s \leq M: \lambda_{t_{i}} \geq 2 K \lambda_{s}\right\}$ then there exists $\gamma_{1}>0$ (depending only on $\eta$ ) such that $k(r)=\max \left\{i: \lambda_{t_{i}} \geq 2 \alpha^{-\frac{1}{2}} K e^{-r}\right\}$ satisfies $k(r) \geq \gamma_{1} r$.

(4) (Vertical displacement) For any $1 \leq i \leq k$ and $\left(s_{1}, \ldots, s_{t_{i}-1}\right)$ there are collections $\mathcal{P}_{1, i}$ and $\mathcal{P}_{2, i}$ as in Claim 1 (with corresponding symbols $\left.s_{t_{i}}^{1}, s_{t_{i}}^{2}\right)$ for which the admissible curves $\varphi^{t_{i}}\left(\omega_{s_{1}, \ldots, s_{t_{i}}^{1}}\right)$ and $\varphi^{t_{i}}\left(\omega_{s_{1}, \ldots, s_{t_{i}}^{2}}\right)$ satisfy

$$
\mid \varphi^{t_{i}}\left(\omega_{s_{1}, \ldots, s_{t_{i}}-1, s_{t_{i}}^{1}}\right)(\theta)-\varphi^{t_{i}}\left(\omega_{s_{1}, \ldots, s_{t_{i}}-1, s_{t_{i}}^{2}}(\theta) \mid \geq \frac{\alpha}{100} \quad \text { for all } \theta \in(0,1]\right.
$$


and, consequently, for any $\left(s_{t_{i}}+1, \ldots, s_{M}\right) \in S^{M-t_{i}}$

$\left|\varphi^{M}\left(\omega_{s_{1}, \ldots, s_{t_{i}}-1, s_{t_{i}}^{1}, s_{t_{i}}+1, \ldots, s_{M}}\right)(\theta)-\varphi^{M}\left(\omega_{s_{1}, \ldots, s_{t_{i}}-1, s_{t_{i}}^{1}, s_{t_{i}}+1, \ldots, s_{M}}\right)(\theta)\right| \geq 4 \sqrt{\alpha} e^{-(r-2)}$.

Now we are in a position to finish the proof of Proposition 4.1, By a small abuse of notation, we write $s_{t_{i}}(\theta) \in \mathcal{P}_{1, i}$ meaning that $\omega_{s_{i}}(\theta) \in \mathcal{P}_{1, i}$, and analogously for the collection $\mathcal{P}_{2, i}$. In fact, one can combine Claim 1 with property (4) above to show that $\operatorname{Leb}\left(\theta \in(0,1]: \hat{Y}_{M}(\theta) \notin \hat{J}(r-2)\right)$ is given by

$$
\begin{aligned}
& \sum_{s_{1} \in S} \sum_{\left(s_{2}, \ldots, s_{M-1}\right) \in S^{M-1}} \operatorname{Leb}\left(\theta \in \omega_{s_{1}, \ldots, s_{M}}: \hat{Y}_{M}(\theta) \notin \hat{J}(r-2)\right) \\
& \quad \geq \operatorname{Leb}\left(\theta: s_{1}(\theta) \in \mathcal{P}_{1,1}\right)+\operatorname{Leb}\left(\theta: s_{1}(\theta) \notin \mathcal{P}_{1,1} \text { and } \hat{Y}_{M}(\theta) \notin \hat{J}(r-2)\right),
\end{aligned}
$$

since admissible segments over $\mathcal{P}_{1,1}$ and $\mathcal{P}_{2,1}$ correspond to vertically displaced admissible curves when mapped by $\varphi^{M}$ and $\mu\left(\bigcup_{\omega \in \mathcal{P}_{1,1}} \omega\right) \leq \mu\left(\bigcup_{\omega \in \mathcal{P}_{2,1}} \omega\right)$. Analogously, the second term in the right hand-side above satisfies

$$
\begin{aligned}
\operatorname{Leb}(\theta \in(0,1]: & \left.s_{1}(\theta) \notin \mathcal{P}_{1,1} \text { and } \hat{Y}_{M}(\theta) \notin \hat{J}(r-2)\right) \\
& =\sum_{s_{1} \notin \mathcal{P}_{1,1}} \sum_{s_{2}, \ldots, s_{t_{2}}-1} \sum_{s_{t_{2}} \notin \mathcal{P}_{1,2}} \sum_{s_{t_{2}+1}, \ldots, s_{M}} \operatorname{Leb}\left(\theta: \hat{Y}_{M}(\theta) \notin \hat{J}(r-2)\right) \\
& +\sum_{s_{1} \notin \mathcal{P}_{1,1}} \sum_{s_{2}, \ldots, s_{t_{2}-1}} \sum_{s_{t_{2}} \in \mathcal{P}_{1,2}} \sum_{s_{t_{2}+1, \ldots, s_{M}} \operatorname{Leb}\left(\theta: \hat{Y}_{M}(\theta) \notin \hat{J}(r-2)\right)} \\
& \geq \sum_{s_{1} \notin \mathcal{P}_{1,1}} \sum_{s_{t_{2}} \notin \mathcal{P}_{1,2}} \operatorname{Leb}\left(\theta: s_{1}(\theta) \notin \mathcal{P}_{1,1} \text { and } s_{t_{2}}(\theta) \notin \mathcal{P}_{1,2}\right) \\
& +\operatorname{Leb}\left(\theta: s_{1}(\theta) \notin \mathcal{P}_{1,1} \text { and } s_{t_{2}}(\theta) \in \mathcal{P}_{1,2} \text { and } \hat{Y}_{M}(\theta) \notin \hat{J}(r-2)\right)
\end{aligned}
$$

Proceeding recursively we obtain that

$$
\begin{aligned}
\operatorname{Leb}\left(\theta: \hat{Y}_{M}(\theta)\right. & \notin \hat{J}(r-2)) \\
& \geq \operatorname{Leb}\left(\theta: s_{1}(\theta) \in \mathcal{P}_{1,1}\right)+\operatorname{Leb}\left(\theta: s_{1}(\theta) \notin \mathcal{P}_{1,1} \text { and } s_{t_{2}}(\theta) \in \mathcal{P}_{1,2}\right) \\
& +\operatorname{Leb}\left(\theta: s_{1}(\theta) \notin \mathcal{P}_{1,1} \text { and } s_{t_{2}}(\theta) \notin \mathcal{P}_{1,2} \text { and } s_{t_{3}}(\theta) \in \mathcal{P}_{1,3}\right) \\
& +\ldots \\
& +\operatorname{Leb}\left(\theta: s_{t_{i}}(\theta) \notin \mathcal{P}_{1, i}, \forall 1 \leq i \leq k(r)-1 \text { and } s_{t_{k(r)}}(\theta) \in \mathcal{P}_{1, k}\right),
\end{aligned}
$$

proving Leb $\left(\theta \in(0,1]: \hat{Y}_{M}(\theta) \in \hat{J}(r-2)\right) \leq \operatorname{Leb}\left(\theta: s_{t_{i}}(\theta) \notin \mathcal{P}_{1, i}, \forall 1 \leq i \leq k(r)\right)$.

Hence, to finish the proof it is enough to prove that the previous right hand side decreases exponentially fast on $r$. Since $\varphi(\theta, x)=(\tilde{g}(\theta), \tilde{f}(\theta, x))$ where $\|g-\tilde{g}\|_{C^{3}}<\varepsilon$ and $g$ is piecewise linear satisfying $\left|g^{\prime}\right| \geq d$ then it follows from Lemmas 3.1 and 3.2 that

$$
\begin{aligned}
\frac{\operatorname{Leb}\left(\omega_{\left(s_{1}, s_{2}, \ldots, s_{k+\ell}\right)}\right)}{\operatorname{Leb}\left(\omega_{\left(s_{1}, s_{2}, \ldots, s_{k}\right)}\right)} & \leq \frac{\operatorname{Leb}\left(\tilde{g}^{k}\left(\omega_{\left(s_{1}, s_{2}, \ldots, s_{k+\ell}\right)}\right)\right)}{\operatorname{Leb}\left(\tilde{g}^{k}\left(\omega_{\left(s_{1}, s_{2}, \ldots, s_{k}\right)}\right)\right)} \exp \left(\frac{d \varepsilon}{(d-1)(d-\varepsilon)^{2}}\right)^{2} \\
& =\operatorname{Leb}\left(\omega_{\left(s_{k+1}, s_{k+2}, \ldots, s_{k+\ell}\right)}\right) \exp \left(\frac{d \varepsilon}{(d-1)(d-\varepsilon)^{2}}\right)^{2}
\end{aligned}
$$


and consequently

$\operatorname{Leb}\left(\omega_{\left(s_{1}, \ldots, s_{k+\ell}\right)}\right) \leq \operatorname{Leb}\left(\omega_{\left(s_{1}, \ldots, s_{k}\right)}\right) \operatorname{Leb}\left(\omega_{\left(s_{k+1}, \ldots, s_{k+\ell}\right)}\right) \exp \left(\frac{d \varepsilon}{(d-1)(d-\varepsilon)^{2}}\right)^{2}$

for any $k+\ell \geq 1$ and sequence $\left(s_{1}, s_{2}, \ldots, s_{k+\ell}\right) \in S^{k+\ell}$. Thus if $\varepsilon$ is small, the previous estimates together with $k(r) \geq \gamma_{1} r$ yield that

$$
\begin{aligned}
\operatorname{Leb}\left(\theta: s_{t_{i}}(\theta) \notin \mathcal{P}_{1, i}, \forall 1 \leq i \leq k(r)\right) & =\sum_{\left\{\left(s_{1}, s_{2}, \ldots, s_{M}\right): s_{t_{i}} \notin \mathcal{P}_{1, i}\right\}} \operatorname{Leb}\left(\omega_{\left(s_{1}, s_{2}, \ldots, s_{M}\right)}\right) \\
& \leq \exp \left(\frac{d \varepsilon}{(d-1)(d-\varepsilon)^{2}}\right)^{2 k(r)} \prod_{j=1}^{k(r)}\left[1-\operatorname{Leb}\left(\mathcal{P}_{1, i}\right)\right] \\
& \leq\left[\exp \left(\frac{2 d \varepsilon}{(d-1)(d-\varepsilon)^{2}}\right)(1-\zeta)\right]^{k(r)} \\
& \leq\left[\exp \left(\frac{2 d \varepsilon}{(d-1)(d-\varepsilon)^{2}}\right)(1-\zeta)\right]^{\gamma_{1} r}
\end{aligned}
$$

This proves that Leb $\left(\theta \in(0,1]: \hat{Y}_{M}(\theta) \in \hat{J}(r-2)\right)$ decreases exponentially fast in $r$ provided that $\varepsilon>0$ is small, and finishes the proof of the proposition.

Observe that our hypothesis yield that the base map is (semi)-conjugated to the on-sided shift with alphabet $S$. Let us point out that a much simpler argument would lead the same result above provided if the $\mu$ is Bernoulli, that is, defined by mass distribution.

4.2. Positive Lyapunov exponents. We are now in a position to prove Theorem A similarly to [Vi97]. First we consider the skew-product $\varphi_{\alpha}$. Let $\gamma \in(0,1)$ be arbitrary and fixed. For any integer $n \geq 1$ set $m=[\sqrt{n}]$ and $\ell=m-M$, where [.] stands as before for the integer part. Given an admissible curve $\hat{Y}$ and $v \in \mathbb{R}^{2}$ non-colinear with $\partial / \partial x$ there is $C>0$ so that $\left\|D \varphi_{\alpha}^{n}(\hat{Y}(\theta)) v\right\| \geq C\left|\left(g^{n}\right)^{\prime}(\theta)\right| \geq C d^{n}$ grows exponentially fast. Hence, it remains to estimate the derivative

$$
\left\|D \varphi_{\alpha}^{n}(\hat{Y}(\theta)) \frac{\partial}{\partial x}\right\|=\prod_{j=0}^{n-1}\left|\frac{\partial f}{\partial x}\left(\hat{Y}_{j}(\theta)\right)\right|,
$$

where the later product can be estimated according to the returns near the critical region using Proposition [3.5. We will say that $1 \leq \nu \leq n$ is a deep return for $\theta$ if $\theta \in \omega$ for some partition element $\omega \in \mathcal{P}^{(\nu+\ell)}$ satisfying $\varphi^{\nu}(\hat{Y} \mid \omega) \cap((0,1] \times J(m)) \neq \emptyset$. We will say that $1 \leq \nu \leq n$ is a regular return for $\theta$ if $\theta \in \omega$ where $\omega \in \mathcal{P}^{(\nu+\ell)}$ satisfies $\varphi^{\nu}\left(\left.\hat{Y}\right|_{\omega}\right) \cap((0,1] \times J(0)) \neq \emptyset$ and $\varphi^{\nu}\left(\left.\hat{Y}\right|_{\omega}\right) \cap((0,1] \times J(m))=\emptyset$. In this case we set the return depth $r_{\nu}(\theta)=$ $\min \left\{r<m: \varphi^{\nu}\left(\left.\hat{Y}\right|_{\omega}\right) \cap((0,1] \times J(r)) \neq \emptyset\right\}$. Observe that the function $r_{\nu}(\cdot)$ is constant on the elements of the partition $\mathcal{P}^{(\nu+\ell)}$. Moreover, since $\varphi^{\nu}\left(\left.\hat{Y}\right|_{\omega}\right)$ is a curve with slope smaller or equal to $\alpha$ and horizontal length smaller or equal to $16^{-\frac{\sqrt{n}}{2}}$, if $\nu$ is a deep return then $\varphi^{\nu}\left(\left.\hat{Y}\right|_{\omega}\right) \subset((0,1] \times J(m-1))$. 
In consequence, from Corollary 3.9 we get

$$
\begin{aligned}
\operatorname{Leb}(\theta \in & (0,1]: \exists 1 \leq \nu \leq n \text { deep return for } \theta) \\
& \leq n \operatorname{Leb}\left(\theta \in(0,1]: \hat{Y}_{\nu}(\theta) \in((0,1] \times J(m-1))\right) \\
& \leq n C \alpha^{-\frac{1}{4}} e^{-\frac{m}{2}} \\
& \leq \alpha^{-\frac{1}{4}} e^{-\frac{1}{3} \sqrt{n}}
\end{aligned}
$$

for all large $n$. In addition, if $\theta \in(0,1]$ has no deep returns and $1 \leq \nu_{1}<$ $\nu_{2}<\cdots<\nu_{s} \leq n$ are the regular returns for $\theta$ with return depths $r_{1}, \ldots, r_{s}$ respectively, then it follows from the estimates in [Vi97, p. 76] that for all large $n$

$$
\log \left\|D \varphi_{\alpha}^{n}(\hat{Y}(\theta)) \frac{\partial}{\partial x}\right\| \geq 2 c n-\sum_{i \in G_{\theta}} r_{i}(\theta)
$$

where $c=\frac{1}{3} \min \left\{\gamma_{2}, \log \sigma_{2}\right\}>0$ and $G_{\theta}=\left\{1 \leq i \leq s: r_{\nu_{i}}(\theta) \geq\left(\frac{1}{2}-\right.\right.$ $\left.\eta) \log \frac{1}{\alpha}\right\}$. Therefore, if $G_{\theta}(q)=\left\{i: \nu_{i} \equiv q(\bmod m)\right\}($ for $0 \leq q<m)$ we obtain that

$$
\begin{aligned}
\operatorname{Leb}\left(\theta \in(0,1]: \sum_{i \in G_{\theta}} r_{i}(\theta) \geq c n\right) & \leq \sqrt{n} \operatorname{Leb}\left(\theta \in(0,1]: \sum_{i \in G_{\theta}(q)} r_{i}(\theta) \geq \frac{c n}{m}\right) \\
& =\sqrt{n} \sum_{R \geq \frac{c n}{m}} \sum_{\left(\begin{array}{c}
\left.\rho_{1}, \ldots, \rho_{\tau}\right) \\
\sum \rho_{j}=R
\end{array}\right.} \operatorname{Leb}\left(\theta: r_{\nu_{j}}(\theta)=\rho_{j}, \forall j\right) \\
& \leq \sqrt{n} \sum_{R \geq \frac{c n}{m}}\left(\begin{array}{c}
R+\tau \\
\tau
\end{array}\right) \tilde{C}^{\tau} e^{-5 \beta \sum_{j} \rho_{j}},
\end{aligned}
$$

where $\tau$ denotes the number of nonzero depths $r_{j}$. Using that $R \geq \tau\left(\frac{1}{2}-\right.$ $\eta) \log \frac{1}{\alpha}$ we can take $\alpha$ small so that $\frac{(R+\tau) !}{R ! \tau !} \tilde{C}^{\tau} \leq e^{\beta R}$ and since

$$
\operatorname{Leb}\left(\theta \in(0,1]: \sum_{i \in G_{\theta}} r_{i}(\theta) \geq c n\right) \leq \sqrt{n} \sum_{R \geq c \frac{n}{m}} e^{-4 \beta R} \leq e^{-\beta \sqrt{n}}
$$

for all large $n$, it is summable. Borel-Cantelli lemma yields that for Lebesgue almost every $\theta$ the expression $\sum_{i \in G_{\theta}} r_{i}(\theta) \leq c n$ holds for all but finitely many values of $n$. Together with equation (4.1) above and using that $\hat{Y}$ was chosen arbitrary, this proves that $\varphi_{\alpha}$ has only positive Lyapunov exponents, that is,

$$
\liminf _{n \rightarrow \infty} \frac{1}{n} \log \left\|D \varphi_{\alpha}^{n}(\theta, x) v\right\| \geq c
$$

for Lebesgue almost every $(\theta, x)$ and every $v \in \mathbb{R}^{2}$, proving the assertion of Theorem $₫$ for the skew-product $\varphi_{\alpha}$. Now, let $\varphi$ be $\varepsilon$-C $C^{3}$-close to $\varphi_{\alpha}$. For $\varphi$ let the critical region $\mathcal{C}$ be defined by $(\theta, x) \in \mathcal{C}$ if and only $\operatorname{det} D \varphi_{\alpha}(\theta, x)=$ 0 . It is not hard to deduce from the Implicit Function Theorem that $\mathcal{C}$ is a $C^{2}$-smooth curve on each invertibility domain, that is, there exists a function $\eta:(0,1] \rightarrow I_{0}$ that is $C^{2}$-close to zero on each interval $\omega_{i}$ satisfying $\mathcal{C}=\operatorname{graph}(\eta)$. Thus one can make a $C^{2}$ change of coordinates and assume that the critical region $\mathcal{C}$ coincides with the segment $\{x=0\}$. Moreover, since $\partial_{x} f(\theta x)=2 x$ we may assume $\partial_{x} \tilde{f}(\theta, x)=|x| \psi(\theta, x)$ with $\psi$ close to 
2 , behaves like a power of the distance to the critical region. This allows to reproduce the previous argument and to show that $\varphi$ has two positive Lyapunov exponents and finishes the proof of Theorem $\mathrm{A}$

\section{SRB MEASURES AND THEIR STATISTICAL PROPERTIES}

This section is devoted to the study of ergodic properties of these robust nonuniformly expanding transformations. In fact we show that there is a unique SRB measure and prove that it has good statistical properties. Throughout let $\varphi$ be $C^{3}$-close to $\varphi_{\alpha}$ and let $\Lambda$ denote the corresponding attractor. We will say that $\varphi$ is topologically exact if for any open set $U$ there exists $N=N(U) \geq 1$ such that $\varphi^{N}(U) \supset \Lambda$. We say that $\varphi$ is ergodic (with respect to Lebesgue) if all $f$-invariant measurable sets are zero or full Lebesgue measure sets.

Proposition 5.1. The map $\varphi$ is topologically exact and ergodic with respect to Leb.

Proof. Since the proof follows closely [AV02, Theorem C] we will omit the details.

At this point one could use [ArS11] to obtain the existence of the absolutely continuous invariant probability measure. However, to deduce the good statistical properties in Theorem $\mathrm{B}$ and to deal with the critical set $\mathcal{C}$ and discontinuities $\mathcal{D}$ for $\varphi$ (formed by countable vertical segments) we need to estimate the tail of the hyperbolic times c.f. [Al00, Definition 2.5]. Since the arguments follow some now standard arguments we focus on the main ingredients.

Using that $\varphi$ is $C^{3}$-close to $\varphi_{\alpha}$, the critical region $\mathcal{C}$ obtained is a piecewise $C^{2}$-smooth curve where $\mathcal{C}=\{(\theta, x): \operatorname{det} D \varphi(\theta, x)=0\}$. Hence we may assume that $\mathcal{C}$ coincides with the segment $\{x=0\}$ and that $\varphi$ behaves like a power of a distance to the critical region along the invariant vertical foliation: there exists $B \geq 1, \beta>0$ so that for all $(\theta, x) \in(0,1] \times I_{0}$ and all $v \in \mathbb{R}^{2}$

$$
\text { (C1) } \frac{1}{B} \operatorname{dist}((\theta, x), \mathcal{C}) \leq \frac{\|D \varphi(\theta, x) v\|}{\|v\|}
$$

and for all points with $\operatorname{dist}\left(\left(\theta_{1}, x_{1}\right),\left(\theta_{2}, x_{2}\right)\right)<\operatorname{dist}\left(\left(\theta_{1}, x_{1}\right), \mathcal{C}\right) / 2$

$$
\begin{aligned}
& \text { (C2) }\left|\log \left\|D \varphi\left(\theta_{1}, x_{1}\right)^{-1}\right\|-\log \left\|D \varphi\left(\theta_{2}, x_{2}\right)^{-1}\right\|\right| \leq B \frac{\operatorname{dist}\left(\left(\theta_{1}, x_{1}\right),\left(\theta_{2}, x_{2}\right)\right)}{\operatorname{dist}\left(\left(\theta_{1}, x_{1}\right), \mathcal{C}\right)^{\beta}} \\
& \text { (C3) }|\log | \operatorname{det} D \varphi\left(\theta_{1}, x_{1}\right)-\log \left|\operatorname{det} D \varphi\left(\theta_{2}, x_{2}\right)\right| \mid \leq B \frac{\operatorname{dist}\left(\left(\theta_{1}, x_{1}\right),\left(\theta_{2}, x_{2}\right)\right)}{\operatorname{dist}\left(\left(\theta_{1}, x_{1}\right), \mathcal{C}\right)^{\beta}} .
\end{aligned}
$$

This will be used to control recurrence to the critical region $\mathcal{C}$. A first step to deduce stretched-exponential decay of correlations using the machinery developed in You98, ALP05, Gou06 we need to obtain non-uniform expansion together with slow recurrence condition to both the critical region $\mathcal{C}$. Notice that

$\liminf _{n \rightarrow \infty} \frac{1}{n} \sum_{j=0}^{n-1} \log \left\|D \varphi\left(\varphi^{j}(\theta, x)\right)^{-1}\right\|^{-1}=\liminf _{n \rightarrow \infty} \frac{1}{n} \log \left\|D \varphi^{n}(\theta, x) \frac{\partial}{\partial x}\right\| \geq c>0$ 
and

$$
(\forall \varepsilon>0)(\exists \delta>0) \quad \limsup _{n \rightarrow \infty} \frac{1}{n} \sum_{j=0}^{n-1}-\log \operatorname{dist}_{\delta}\left(\varphi^{j}(\theta, x), \mathcal{C}\right)<\varepsilon
$$

holds for Lebesgue almost every $(\theta, x)$, where $\operatorname{dist}_{\delta}(z, \mathcal{C})=\operatorname{dist}(z, \mathcal{C})$ if $\operatorname{dist}(z, \mathcal{C})<\delta$ and $\operatorname{dist}_{\delta}(z, \mathcal{C})=0$ otherwise. In fact, on the one hand

$$
\begin{aligned}
\mathcal{E}_{v}(\theta, x) & :=\min \left\{N \geq 1: \frac{1}{n} \sum_{j=0}^{n-1} \log \left\|D \varphi\left(\varphi^{j}(\theta, x)\right)^{-1}\right\|^{-1} \geq c, \text { for all } n \geq N\right\} \\
& \leq \min \left\{N \geq 1: r_{j}(\theta, x) \leq c n(\forall 1 \leq j \leq n) \text { and } \sum_{j=0}^{n-1} r_{i}(\theta, x) \leq c n(\forall n \geq N)\right\},
\end{aligned}
$$

is well defined and finite for Lebesgue almost every point. Furthermore, it follows that $\operatorname{Leb}\left((\theta, x) \in(0,1] \times I_{0}: \mathcal{E}_{v}(\theta, x) \geq n\right) \leq C e^{-\gamma \sqrt{n}}$ for all large $n$. On the other hand, given $\varepsilon, \delta>0$ consider the Lebesgue almost everywhere well defined function

$\mathcal{R}_{v, \varepsilon, \delta}(\theta, x):=\min \left\{N \geq 1: \sum_{j=0}^{n-1}-\log \operatorname{dist}_{\delta}\left(\varphi^{j}(\theta, x), \mathcal{C}\right)<\varepsilon n\right.$ for all $\left.n \geq N\right\}$.

Notice that if $\delta=\left(\frac{1}{2}-2 \eta\right) \log \frac{1}{\alpha}$ then $\sum_{j=0}^{n-1}-\log \operatorname{dist}_{\delta}\left(\varphi^{j}(\theta, x), \mathcal{C}\right) \leq \sum_{j=0}^{n-1} r_{j}(\theta, x)$ for all $(\theta, x)$. Hence, the same large deviations argument of Section 4 yield that there exists $\gamma(\varepsilon)>0$ such that

$$
\left.\operatorname{Leb}\left((\theta, x): \mathcal{R}_{v, \varepsilon, \delta}(\theta, x) \geq n\right) \leq \operatorname{Leb}(\theta, x): \sum_{j=0}^{n-1} r_{j}(\theta, x)>\varepsilon n\right) \leq C e^{-\gamma(\varepsilon) \sqrt{n}}
$$

for all large $n$. In consequence, this proves that for any $\varepsilon, \delta>0$ there exists $\tilde{\gamma}(\varepsilon)=\min \{\gamma, \gamma(\varepsilon)\}$ such that

$$
\operatorname{Leb}\left((\theta, x) \in(0,1] \times I_{0}: \mathcal{E}_{v}(\theta, x) \geq n \text { or } \mathcal{R}_{v, \varepsilon, \delta}(\theta, x) \geq n\right) \leq C e^{-\tilde{\gamma}(\varepsilon) \sqrt{n}}
$$

for all large $n$. On the one hand, despite the discontinuities, it follows from the Markov assumption and bounded distortion Lemma 3.2 that for any partition element $\omega \in \mathcal{P}^{n}$ the map $\left.g^{n}\right|_{\omega}: \omega \rightarrow(0,1]$ has bounded distortion and the backward contraction property $d\left(g^{n-j}(y), g^{n-j}(z)\right) \leq(d-$ $\varepsilon)^{-j} d\left(g^{n}(y), g^{n}(z)\right)$ for all $0 \leq j \leq n$ and $y, z \in \omega$. In consequence, $g$ admits a unique absolutely continuous invariant probability measure $\eta$ equivalent to Lebesgue. Therefore, since $\varphi$ is a skew product, if $\mathcal{R}_{h, \varepsilon, \delta}(\theta, x)=\min \{N \geq$ $1: \sum_{j=0}^{n-1}-\log \operatorname{dist}_{\delta}\left(\varphi^{j}(\theta, x), \mathcal{D}\right)<\varepsilon n$ for all $\left.n \geq N\right\}$ and also $\mathcal{R}_{g, \varepsilon, \delta}(\theta)=$ $\min \left\{N \geq 1: \sum_{j=0}^{n-1}-\log \operatorname{dist}_{\delta}\left(g^{j}(\theta), \pi_{1}(\mathcal{D})\right)<\varepsilon n\right.$ for all $\left.n \geq N\right\}$ then it follows that

$$
\operatorname{Leb}\left((\theta, x): \mathcal{R}_{h, \varepsilon, \delta}(\theta, x)>n\right)=\operatorname{Leb}\left(\theta \in(0,1]: \mathcal{R}_{g, \varepsilon, \delta}(\theta)>n\right)
$$

where $\pi_{1}$ stands for the projection on the $\theta$-coordinate. Furthermore, since $\eta$ is equivalent to Lebesgue and has exponential decay of correlations it yields exponential large deviations for Hölder observables. Thus, we can proceed as in AS13 (building over ALFV11) to obtain an exponential large deviation estimate for (5.1) and, in consequence, sub-exponential tails for the expansion and return time functions. Thus, it follows from Gou06] 
that there exists a $\varphi$-invariant absolutely continuous probability measure $\mu$ with stretched-exponential decay of correlations. In consequence,

Proposition 5.2. There exists a unique $S R B$ measure $\mu$ for $\varphi$. Moreover, the basin of attraction $B(\mu)$ contains Lebesgue almost every point in $\Lambda$.

Proof. Let $\mu$ be the $\varphi$-invariant and ergodic probability measure constructed above. Since $\mu \ll$ Leb then it is clearly an SRB measure because its basin of attraction

$$
B(\mu)=\left\{(\theta, x) \in \Lambda: \frac{1}{n} \sum_{j=0}^{n-1} \delta_{\varphi^{j}(\theta, x)} \rightarrow \mu\right\}
$$

is an $\varphi$-invariant set and a $\mu$-full measure set. Using that $\varphi$ is exact then it follows that $B(\mu)$ has full Lebesgue measure set in $\Lambda$. This also proves uniqueness of the SRB measure and finishes the proof of the proposition.

Finally, it follows from ALFV11 that stretched-exponential decay of correlations imply on stretched-exponential large-deviation bounds and also in the central limit theorem, almost sure invariance principle, local limit theorem and Berry-Esseen theorem. This finishes the proof of Theorem B.

\section{Coexistence of positive And negative Lyapunov exponents FOR GENERIC GENERALIZED VIANA-MAPS}

This section is devoted to the proof of Theorem $\mathrm{C}$ where, in particular, we prove that generic generalized Viana maps have a dense set of points with negative central Lyapunov exponent. We will make use of the density of hyperbolicity for maps of the interval in Theorem 3.4 .

Proof of Theorem $\mathbb{C}$. Let $\mathcal{V}$ be an open set of generalized Viana maps. By construction every $\varphi \in \mathcal{V}$ is conjugated to a skew-product over the same (topological) Markov expanding map $g$. Moreover, using that $g$ has a fixed point $p$ it follows that the map $\varphi$ preserves the fiber over one fixed point $p_{*}=p_{*}(\varphi)$. In other words, one can consider the unimodal map

$$
\varphi_{p_{*}}(\cdot)=\varphi\left(p_{*}, \cdot\right):\left\{p_{*}\right\} \times I_{0} \rightarrow\left\{p_{*}\right\} \times I_{0} .
$$

By topological conjugacy the continuation of the point $p_{*}(\varphi)$ is well defined for all $\varphi$. Using Theorem 3.4, we deduce that there exists an open and dense set $\mathcal{A} \subset \mathcal{V}$ such that for any $\varphi \in \mathcal{A}$ the corresponding unimodal map $\varphi_{p_{*}}$ is hyperbolic. In consequence, $\varphi_{p_{*}}$ admits a periodic attractor whose the basin of attraction s a full Lebesgue measure set, it is open and dense. Therefore, $\varphi$ has a periodic saddle $P_{*}$ on the fiber over $p_{*}$.

Now, notice that the pre-orbit $\mathcal{O}^{-}\left(p_{*}\right)=\left\{\theta \in(0,1]:(\exists n \geq 1) g^{n}(\theta)=\right.$ $\left.p_{*}\right\}$ is dense in $(0,1]$ and so the set $D=\left\{(\theta, x): \theta \in \mathcal{O}^{-}\left(p_{*}\right), x \in I_{0}\right\}$ is dense in the attractor $\Lambda(\varphi)$. Since the fibered maps are non-singular with respect to Lebesgue then there exists a dense subset of points in $D$ that are forward asymptotic to the saddle point $P_{*}$ and, consequently, have one negative Lyapunov exponent as claimed in part (1). Since the second assertion follows directly from Theorem A this finishes the proof of the theorem. 


\section{SRB MEASURES FOR SKEW-PRODUCTS OF FIBERED NEARBY \\ HYPERBOLIC INTERVAL MAPS}

The main purpose of this section is to prove Theorem $\mathrm{D}$ for fibered maps obtained by perturbation of hyperbolic interval maps, which is of independent interest. So, let $T: I \rightarrow I$ be a hyperbolic $C^{3}$-interval map with negative Schwarzian derivative in the interval $I$ and $S: X \rightarrow X$ be a continuous map that admits a unique SRB measure $\mu_{S}$, where $X$ is a compact Riemannian manifold. Consider the skew product

$$
\psi:(x, y) \mapsto(S(x), T(y)+a(x))
$$

associated to a $C^{3}$-function $a: X \rightarrow \mathbb{R}$. Given a point $x \in X$, consider the iteration $\psi_{x}^{n}=\psi_{T^{n-1}(x)} \circ \cdots \circ \psi_{T(x)} \circ \psi_{x}$. First we proceed to prove that if $\|a\|_{C^{3}}<\varepsilon$, for a small $\varepsilon$, then the skew product $\psi$ admits a trapping region $\mathcal{U}$. Moreover we prove that the attractor $\mathcal{G} \subset \mathcal{U}$ supports a unique $\mathrm{SRB}$ measure $\nu$, ergodic, whose basin of attraction contains Lebesgue almost every point in $\mathcal{U}$.

By hyperbolicity, $T$ has a a finite number of periodic attracting points $\left\{p_{i}\right\}$ of period $\pi\left(p_{i}\right) \geq 1$ and $I=K \cup\left(\cup_{i} B\left(p_{i}\right)\right)$, where $B\left(p_{i}\right)$ denotes the topological basin of attraction of $p_{i}$ and $K$ is an invariant Cantor set such that $\left.T\right|_{K}$ is expanding. Since hyperbolicity is $C^{r}$-open and dense in the interval (see $\mathrm{KSvS07a}$ ) and $\alpha$ is assumed small, by structural stability all interval maps $T_{x}=T+a(x)$ are hyperbolic and topologically conjugated to $T$ : for every $x \in X$ there exists an homeomorphism $h_{x}$ that is $C^{0}$-close to identity, varies continuously with $x$ and $T_{x} \circ h_{x}=h_{x} \circ T$. In consequence, $p_{i}^{x}=h_{x}\left(p_{i}\right)$ is an attracting periodic point of period $\pi\left(p_{i}\right)$ and $K_{x}=h_{x}(K)$ is an invariant expanding Cantor set for the interval map $T_{x}$. We first prove that similar features are inherited by the skew-product $\psi$.

Proposition 7.1. If $\varepsilon>0$ is small, there exists $N \geq 1$ and an open set $U \subset I$ obtained as finite union of open intervals and $c>0$ so that $\mathcal{U}=X \times U$ is positively $\psi^{N}$-invariant and

$$
\limsup _{n \rightarrow \infty} \frac{1}{n} \log \left\|D \psi^{n}(x, y) \frac{\partial}{\partial y}\right\| \leq-c<0
$$

for all $(x, y) \in \mathcal{U}$. Moreover, there exists a small open neighbourhood $V$ of the critical points for $T$ and $K \geq 1$ such that $\varphi^{K}(X \times V) \subset \mathcal{U}$.

Proof. Given any $i$, since $p_{i}$ is a periodic atractor for $T$ then using the uniform continuity of the derivative there exists $\hat{\lambda}_{i}<1$ and an open neighborhood $\mathcal{U}_{i}$ of the forward orbit of $p_{i}$ such that $T^{\pi\left(p_{i}\right)}\left(U_{i}\right) \subsetneq U_{i}$ and $\left|\left(T^{\pi\left(p_{i}\right)}\right)^{\prime}(y)\right| \leq$ $\hat{\lambda}_{i}^{\pi\left(p_{i}\right)}<1$ for all $y \in U_{i}$. Furthermore, if $\varepsilon$ is small, the fact that $\| T-$ $T_{x} \|_{C^{3}}<\varepsilon$ there exists $\hat{\lambda}_{i}<\lambda_{i}<1$ satisfying $T_{x}^{\pi\left(p_{i}\right)}\left(U_{i}\right) \subsetneq U_{i}$ and $\left|\left(T_{x}^{\pi\left(p_{i}\right)}\right)^{\prime}(y)\right| \leq$ $\lambda_{i}^{\pi\left(p_{i}\right)}<1$ for all $y \in U_{i}$.

Set $N=\prod_{i} \pi\left(p_{i}\right)$. On the one hand the later proves that the open set $U$ obtained by the finite union of the open intervals $U_{i}$ is $T_{x}^{N}$-invariant for all $x \in X$ and, consequently, the set $\mathcal{U}=X \times U$ is positively $\psi^{N}$-invariant. On the other hand, if $\lambda$ is chosen such that $\lambda_{i}<\lambda<1$ for all $i$ then by the chain rule $\left|\left(T_{x}^{N}\right)^{\prime}(y)\right| \leq \lambda^{N}$ for all $y \in U$ and $x \in X$. Now we observe that 
if $n=q N+r$ with $q \in \mathbb{N}_{0}$ and $0 \leq r \leq N-1$ then

$$
\begin{aligned}
\left\|D \psi^{n}(x, y) \frac{\partial}{\partial y}\right\| & =\left|\left(T_{S^{n-1}(x)} \circ \cdots \circ T_{S(x)} \circ T_{x}\right)^{\prime}(y)\right| \\
& =\left|\left(T_{S^{q N}(x)}^{r}\right)^{\prime}\left(T_{x}^{q N}(y)\right)\right| \prod_{j=0}^{q-1}\left|\left(T_{S^{j N}(x)}^{N}\right)^{\prime}\left(T_{x}^{j N}(y)\right)\right| \\
& \leq\left\|T^{\prime}\right\|_{\infty}^{N} \lambda^{-N} \lambda^{n}
\end{aligned}
$$

for all $(x, y) \in \mathcal{U}$. Since the later implies that

$$
\limsup _{n \rightarrow \infty} \frac{1}{n} \log \left\|D \psi^{n}(x, y) \frac{\partial}{\partial y}\right\| \leq \log \lambda<0
$$

this finishes the proof of first statement of the proposition.

Now, since $T$ has negative Schwarzian derivative one can use a theorem by Singer to deduce that the orbit of any critical point belongs to the topological basin of attraction of some periodic attractor. Since there are finitely many periodic attractors for $T$ then there exists $K \geq 1$ such that $T^{K}(c) \in U$ for every $c$ such that $T^{\prime}(c)=0$. By continuity, there exists an open neighbourhood $V$ of the critical points for $T$ such that $\psi^{K}(X \times V) \subset \mathcal{U}$, provided that $\alpha$ is small enough. This finishes the proof of the proposition.

Remark 7.2. Let us mention that the set $\bigcup_{i}\left\{\left(x, h_{x}\left(T^{j}\left(p_{i}\right)\right)\right): x \in X, 1 \leq\right.$ $\left.j \leq \pi\left(p_{i}\right)\right\} \subset \mathcal{U}$ of curves formed by the continuations of the periodic attractors is not necessarily $\psi$-invariant (e.g. if $a$ is a Morse function it causes implies a transversality condition for admissible curves). An interesting question is to determine if, under some conditions on the function $a$, the positively invariant set $\mathcal{G}=\bigcap_{n \geq 0} \psi^{n}(\mathcal{U})$ support an absolutely continuous SRB measure.

We now describe the asymptotics of Lebesgue almost every point that is mapped in the trapping region $\mathcal{U}$. More precisely we prove the following:

Proposition 7.3. There exists $\varepsilon>0$ such that if $\|a\|_{C^{3}}<\varepsilon$ then there exists an SRB measure $\nu$ for $\psi$, it is hyperbolic and its basin of attraction contains Lebesgue almost every point in some open subset of $\mathcal{U}$.

Proof. Let $\mu_{S}$ denote the unique SRB measure for $S$ and $\varepsilon$ and the trapping region $\mathcal{U}=X \times U$ as in Proposition 7.1. Take $y_{0} \in U$ arbitrary and consider the sequence of probability measures given by

$$
\nu_{n}=\frac{1}{n} \sum_{j=0}^{n-1} \psi_{*}^{j}\left(\mu_{S} \times \delta_{y_{0}}\right), \quad n \geq 1 .
$$

By construction, $x_{0} \in U_{i}$ for some $i$ and thus belongs to the topological basin of atraction of $p_{i}$ with respect to the polynomial $T$.

We claim that $\left(\nu_{n}\right)_{n}$ is convergent to an $\varphi$-invariant, ergodic probability measure $\nu$ whose basin of attraction covers Lebesgue almost every point in the open set $X \times U_{i}$. On the one hand, if one considers the projection $\pi_{X}: X \times I \rightarrow X$ on the first coordinate then the sequence $\left(\pi_{X}\right)_{*} \nu_{n}=$ $\frac{1}{n} \sum_{j=0}^{n-1} S_{*}^{j} \mu_{S}=\mu_{S}$ for all $n$. On the other hand, the uniform contraction on $X \times U_{i}$ under iterations by $T_{x}^{n}$ implies that for any continuous observable $G$, 
$\delta>0$ and points $y_{1}, y_{2} \in\{x\} \times U_{i}$ the Birkhoff averages satisfy $\mid \frac{1}{n} \sum_{j=0}^{n-1} G \circ$ $\psi^{j}\left(x, y_{1}\right)-\frac{1}{n} \sum_{j=0}^{n-1} G \circ \psi^{j}\left(x, y_{2}\right) \mid<\delta$ provided that $n$ is large (depending only on $G$ and $\lambda)$. Therefore, the functional $\Psi: C\left(X \times \overline{U_{i}}\right) \rightarrow \mathbb{R}$ given by

$$
\begin{aligned}
\Psi(G) & =\lim _{n \rightarrow \infty} \frac{1}{n} \sum_{j=0}^{n-1} \int \max _{y \in \bar{U}_{i}}\left[G \circ \psi^{j}(x, y)\right] d \mu_{S}(x) \\
& =\lim _{n \rightarrow \infty} \frac{1}{n} \sum_{j=0}^{n-1} \int G \circ \psi^{j}\left(x, y_{0}\right) d \mu_{S}(x) \\
& =\lim _{n \rightarrow \infty} \int G d \nu_{n}
\end{aligned}
$$

is well defined and clearly continuous. By Riesz representation theorem there exists a probability measure $\nu$ with $\operatorname{supp}(\nu) \subset X \times \overline{U_{i}}$ and such that $\Psi(G)=\int G d \nu$ for all continuous function $G$. Furthermore, $\left(\pi_{X}\right)_{*} \nu=\mu_{X}$ and, since $\mu_{X}$ is $S$-invariant and ergodic, the limit (7.2) is almost everywhere constant in $x$ and consequently

$$
\int G d \nu=\lim _{n \rightarrow+\infty} \frac{1}{n} \sum_{j=0}^{n-1} G \circ \psi^{j}(x, y)
$$

for $\mu_{S} \times$ Leb-almost every $(x, y) \in X \times \overline{U_{i}}$. In particular, this proves that $\nu$ is $\psi$-invariant and ergodic, and that Lebesgue almost every $(x, y) \in X \times \overline{U_{i}}$ belongs to the basin of attraction $B(\nu)$ and so $\nu$ is the unique SRB measure supported in $X \times \overline{U_{i}}$.

Hence to finalize the proof of Theorem $\mathrm{D}$ let us assume that $\mu_{S}$ has only non-zero Lyapunov exponents and prove that the $\operatorname{SRB}$ measure $\nu$ is hyperbolic. This holds from the fact that the Lyapunov exponents for $\nu$ coincide with the ones of $\mu_{S}$ together with the fibered Lyapunov exponent, which is negative since it is bounded from above by (7.1) since $\nu$ is supported in $X \times \overline{U_{i}}$. This finishes the proof of the theorem.

Remark 7.4. One final remark is that it is not hard to check from the proof above that there are exactly as many hyperbolic SRB measures supported in $\mathcal{U}$ as the number of periodic attracting points for the hyperbolic interval map $T$. This comes from the fact that the measure $\delta_{y_{0}}$ is associated to a point $y_{0}$ that belongs to the topological basin of attraction of some of the periodic attractors.

Acknowledgments. The author is grateful to J. F. Alves, J. Rivera-Letelier, D. Schnellmann, S. Senti, O. Sester and M. Viana for their comments and inspiring conversations. This work was partially supported by $\mathrm{CNPq}$ and FAPESB.

\section{REFERENCES}

[A100] J. F. Alves. SRB measures for non-hyperbolic systems with multidimensional expansion. Ann. Sci. École Norm. Sup., 33:1-32, 2000.

[ALFV11] J. F. Alves, S. Luzzatto, J. Freitas and S. Vaienti. From rates of mixing to recurrence times via large deviations. Adv. Math. 228:2,1203-1236, 2011. 
[ALP05] J. F. Alves, S. Luzzatto and V. Pinheiro. Markov structures and decay of correlations for non-uniformly expanding dynamical systems. Ann. Inst. H. Poincaré Anal. Non Linéaire, 22, 817-839, 2005.

[AS13] J. F. Alves and D. Schnellmann. Ergodic properties of Viana-like maps with singularities in the base dynamics. Proc. Amer. Math. Soc. (to appear 2013).

[AV02] J. F. Alves and M. Viana. Statistical stability for robust classes with non-uniform expansion. Ergod. Th. Dynam. Sys., 22, 1-32, 2002.

[ArS11] V. Araújo and J. Solano. Absolutely continuous invariant measures for nonexpanding skew products. Preprint arXiv:1111.4540

[AP06] V. Araújo and M.J. Pacífico. Large deviations for non-uniformly expanding maps. J. Statist. Phys., 125:415-457, 2006.

[BC85] M. Benedicks and L. Carleson. On iterations of $1-a x^{2}$ on $(-1,1)$. Annals of Math., 122, 1-25, 1985.

[BDV05] C. Bonatti and L. J. Díaz and M. Viana. Dynamics beyond uniform hyperbolicity. Springer-Verlag, Encyclopaedia of Mathematical Sciences, 2005.

[Bo75] R. Bowen. Equilibrium states and the ergodic theory of Anosov diffeomorphisms, volume 470 of Lect. Notes in Math. Springer Verlag, 1975.

[BR75] R. Bowen and D. Ruelle. The ergodic theory of Axiom A flows. Invent. Math., 29:181-202, 1975.

[BST03] J. Buzzi, O. Sester and M. Tsujii. Weakly expanding skew-products of quadratic maps. Ergod. Th. Dynam. Sys, 28, 245-266, 2003.

[dMvS92] W. de Melo and S. van Strien One dimensional dynamics. Springer Verlag, 1992.

[Gou06] S. Gouëzel. Decay of correlations for nonuniformly expanding maps. Bull. Soc. Math. France, 134, 1-31, 2006.

[Gou07] S. Gouëzel. Statistical properties of a skew product with a curve of neutral points. Ergod. Th. Dynam. Sys, 27:123-151, 2007.

[GS97] J. Graczyk and G. Świątek Generic hyperbolicity in the logistic family. Annals of Math., 146, 1-52, 1997.

[GS12] R. Gao and W. Shen Analytic skew-products of quadratic polynomials over Misiurewicz-Thurston maps . Preprint arXiv:1207.2702

[HM03] T. Hunt and R. MacKay. Anosov parameter values for the triple linkage and a physical system with a uniformly chaotic attractor. Nonlinearity, 16:1499-1510, 2003.

[Jak81] M. Jakobson. Absolutely continuous invariant measures for one-parameter families of one-dimensional maps. Comm. Math. Phys., 81, 39-88, 1981.

[Jo97] M. Jonsson. Dynamical studies in several complex variables. Royal Institute of Technology, Stockholm, 1997.

[KSvS07a] O. Kozlovski, W. Shen and S. van Strien. Density of hyperbolicity in dimension one. Annals of Math. (2), 166, 145-182, 2007.

[Lyu97] M. Lyubich. Dynamics of quadratic polynomials. I, II. Acta Math. 178, 185-247, 247-297, 1997

[Pi11] V. Pinheiro. Zooming measures and inducing schemes. Annales de l'IHP - Analyse Non-Lineaire, v. 28, p. 889-939, 2011.

[MN08] I. Melbourne and M. Nicol, Large deviations for nonuniformly hyperbolic systems. Trans. Amer. Math. Soc., 360, 6661-6676, 2008.

[Sa03] O. Sarig. Existence of Gibbs measures for countable Markov shifts. Proc. Amer. Math. Soc., 131:1751-1758 (electronic), 2003.

[Se99] O. Sester. Hiperbolicité des polynômes fibrés. Bull. Soc. Mat. France, 127, 393-428 1999.

[Sc08] D. Schnellmann. Non-continuous weakly expanding skew-products of quadratic maps with two positive Lyapunov exponents. Ergod. Th. Dynam. Sys., 28, 245-266, 2008.

[Sc09] D. Schnellmann. Positive Lyapunov exponents for quadratic skew-products over a Misiurewicz-Thurston map. Nonlinearity, 22, 2681-2695 , 2009.

[Sm67] S. Smale Differentiable dynamical systems. Bull. Am. Math. Soc., 73, 747-817, 1967. 
[Sm00] S. Smale Mathematical Problems for the Next Century, Mathematics : Frontiers and Perspectives. Amer. Math. Soc., Providence, RI, 271-294, 2000.

[Tsu01] M. Tsujii Fat solenoidal attractors. Nonlinearity, 14 , 1011-1027, 2001.

[Va12] P. Varandas. Non-uniform specification and large deviations for weak Gibbs measures. J. Stat. Phys., 146, 330-358, 2012.

[Vi97] M. Viana. Multidimensional nonhyperbolic attractors. Inst. Hautes Études Sci. Publ. Math., 85:63-96, 1997.

[Vo11] D. Volk. Persistent massive attractors of smooth maps. Preprint arXiv:1108.5330. [You98] L.-S. Young. Statistical properties of dynamical systems with some hyperbolicity. Annals of Math., 147:585-650, 1998.

Departamento de Matemática, Universidade Federal da Bahia, Av. Ademar DE Barros s/n, 40170-110 Salvador, Brazil.

E-mail address: paulo.varandas@ufba.br, http://www.pgmat.ufba.br/varandas/ 